\title{
DERIVED EQUIVALENCES BETWEEN SYMMETRIC SPECIAL BISERIAL ALGEBRAS
}

\author{
TAKUMA AIHARA
}

\begin{abstract}
The notion of mutation plays crucial roles in representation theory of algebras. Two kinds of mutation are well-known: tilting/silting mutation and quivermutation. In this paper, we focus on tilting mutation for symmetric algebras. Introducing mutation of SB quivers, we explicitly give a combinatorial description of tilting mutation of symmetric special biserial algebras. As an application, we generalize Rickard's star theorem. We also introduce flip of Brauer graphs and apply our results to Brauer graph algebras.
\end{abstract}

\section{INTRODUCTION}

Tilting mutation, which is a special case of silting mutation [AI], was introduced by Riedtmann-Schofield [RS] and Happel-Unger [HU] to investigate the structure of the derived category. For example, Bernstein-Gelfand-Ponomarev reflection functors BGP, Auslander-Platzeck-Reiten tilting modules APR and Okuyama-Rickard tilting complexes [Q, R2 are special cases of tilting mutation. In the case that a given algebra is symmetric, tilting mutation yields infinitely many tilting complexes. From Morita theoretic viewpoint of derived categories [R1], tilting complexes are extremely important complexes since they give rise to derived equivalences which preserve many homological properties.

The following problem is naturally asked:

Problem 1.1. Give an explicit description of the endomorphism algebra of a tilting complex given by tilting mutation.

In this paper we give a complete answer to this problem for symmetric special biserial algebras, which is one of the important classes of algebras in representation theory. Some of special biserial algebras were first studied by Gelfand-Ponomarev [GP], and also naturally appear in modular representation theory of finite groups Alp, E. Moreover such an algebra is always representation-tame and the classification of all indecomposable modules of such an algebra was provided in [WW, $\overline{B R}$. The derived equivalence classes of special biserial algebras were also discussed in [Al, BHS, [K, KR, , SZ].

To realize our goal, we start with describing symmetric special biserial algebras in terms of combinatorial data, which we call $S B$ quivers. Moreover we will study symmetric special biserial algebras from graph theoretic viewpoint, which is discribed by Brauer graphs. Indeed, we have the result below (see Lemma 2.3 and Proposition 5.5):

Proposition 1.2. There exist one-to-one correspondences among the following three classes:

Date: October 22, 2018.

Key words and phrases. special biserial algebra, mutation of SB quivers, tilting mutation, Brauer graph algebra, flip of Brauer graphs.

2010 Mathematics Subject Classification. Primary 16G10; Secondary 16G20, 18E30, 20 C05. 
(1) Symmetric special biserial algebras;

(2) Special quivers with cycle-decomposition (SB quivers);

(3) Brauer graphs.

We introduce mutation of $S B$ quivers (see Definition 3.2, 3.5 and 3.8), which is similar to Fomin-Zelevinsky quiver-mutation [FZ]. Moreover we will show that mutation of SB quivers corresponds to a certain operation on Brauer graphs, which we call flip and is a generalization of mutation/flip of Brauer trees introduced in [A, [KZ].

The main theorem of this paper is the following:

Theorem 1.3 (Theorem 3.1 and Theorem 5.8). The following three operations are compatible each other:

(1) Tilting mutation of symmetric special biserial algebras;

(2) Mutation of $S B$ quivers;

(3) Flip of Brauer graphs.

We note that certain special cases of the compatibility of (1) and (3) in Theorem 1.3 were given by [K, $\mathrm{An}]$ (see Remark 5.9).

The strategy of our proof of Theorem 1.3 is, by using the fact that the property of being a symmetric special biserial algebra is derived invariant, to resolve into the calculation of numerical invariants but not directly calculate endomorphism algebras. Thus, our proof is simpler than that of $[\mathrm{K}, \mathrm{An}]$.

As an application of Theorem 1.3, we generalize "Rickard's star theorem" for Brauer tree algebras, which gives nice representatives of Brauer tree algebras up to derived equivalence [R2, (M]. We introduce Brauer double-star algebras, as the corresponding class for Brauer tree algebras (see also [K, $\mathrm{KR}, \mathrm{Ro}]$ ), and prove the following (see Section 5.3 for the details):

Theorem 1.4 (Theorem 5.10). Any Brauer graph algebra is derived equivalent to a Brauer double-star algebra whose Brauer graph has the same number of the edges and the same multiplicities of the vertices.

Our proof of Theorem 1.4 is given in terms of the corresponding symmetric special biserial algebras (Theorem 4.1). As an application of Theorem 1.4, we deduce Rickard's star theorem (Corollary 5.12).

This paper is organized as follows: In Section 2, we recall the definition of special biserial algebras and introduce the notion of SB quivers. It is seen that the class of symmetric special biserial algebras coincides with that of SB quivers (Lemma 2.3), which plays an important role in this paper. Moreover, we study tilting complexes introduced by Okuyama and Rickard. In Section 3, mutation of SB quivers is introduced. Theorem 1.3 is also stated. In Section 4, applying Theorem 1.3, we establish a method for reducing some cycles (Method 4.2). It is observed that this method implies 'reduction' theorem for symmetric special biserial algebras. In Section 5, we introduce flip of Brauer graphs and see that it is compatible with tilting mutation of Brauer graph algebras (Theorem [5.8). Moreover, Theorem 1.4 is also obtained. In Section 6, we shall prove Theorem 1.3.

Acknowledgement. The author would like to express his deep gratitude to Osamu Iyama who read the paper carefully and gave a lot of helpful comments and suggestions. The author would also like to thank the referees for carefully reading the paper and for giving constructive comments. 


\section{SymmetriC SPECIAL BISERIAL ALGEBRAS}

This section is devoted to introducing the notion of SB quivers. We will give a relationship between symmetric special biserial algebras and SB quivers. Moreover we study tilting mutation, which is a special case of silting mutation introduced by [AI].

Throughout this paper, we use the following notation.

Notation. Let $A$ be a finite dimensional algebra over an algebraically closed field $k$.

(1) We always assume that $A$ is basic and indecomposable.

(2) We often write $A=k Q / I$ where $Q$ is a finite quiver with relations $I$. The sets of vertices and arrows of $Q$ are denoted by $Q_{0}$ and $Q_{1}$, respectively.

(3) We denote by mod $A$ the category of finitely generated right $A$-modules. A simple (respectively, indecomposable projective) $A$-module corresponding to a vertex $i$ of $Q$ is denoted by $S_{i}$ (respectively, by $P_{i}$ ). We always mean that a module is finitely generated.

A quiver of the form $\longrightarrow \bullet \longrightarrow \cdots \longrightarrow$ with $n$ arrows is called an $n$-cycle (for simplicity, cycle). We mean 1-cycle by loop.

Let us start with introducing SB quivers.

Definition 2.1. We say that a finite connected quiver $Q$ is special if any vertex $i$ of $Q$ is the starting point of at most two arrows and also the end point of at most two arrows. For a special quiver $Q$ with at least one arrow, a set $\mathrm{C}=\left\{C_{1}, C_{2}, \cdots, C_{v}\right\}$ of cycles in $Q$ with a function mult : $\mathrm{C} \rightarrow \mathbb{N}$ is said to be a cycle-decomposition if it satisfies the following conditions:

(1) Each $C_{\ell}$ is a subquiver of $Q$ with at least one arrow such that $Q_{0}=\left(C_{1}\right)_{0} \cup \cdots \cup\left(C_{v}\right)_{0}$ and $Q_{1}=\left(C_{1}\right)_{1} \amalg \cdots \amalg\left(C_{v}\right)_{1}$ : For any $\alpha \in Q_{1}$, we denote by $C_{\alpha}$ a unique cycle in C which contains $\alpha$.

(2) Any vertex of $Q$ belongs to at most two cycles.

(3) $\operatorname{mult}\left(C_{\ell}\right)>1$ if $C_{\ell}$ is a loop.

A $S B$ quiver is a pair $(Q, C)$ of a special quiver $Q$ and its cycle-decomposition C.

Let $(Q, \mathrm{C})$ be a SB quiver. For each cycle $C$ in $\mathrm{C}$, we call mult $(C)$ the multiplicity of $C$.

For any arrow $\alpha$ of $Q$, we denote by na $(\alpha)$ a unique arrow $\beta$ such that $\bullet \stackrel{\alpha}{\longrightarrow} \bullet \stackrel{\beta}{\longrightarrow} \bullet$ appears in $C_{\alpha}$.

We construct a finite dimensional algebra from a SB quiver.

Definition 2.2. Let $(Q, \mathrm{C})$ be a $\mathrm{SB}$ quiver. An ideal $I_{(Q, \mathrm{C})}$ of $k Q$ is generated by the following three kinds of elements:

(i) $\left(\alpha_{t} \alpha_{t+1} \cdots \alpha_{t+s-1}\right)^{m} \alpha_{t}$ for each cycle $C$ in $\mathrm{C}$ of the form

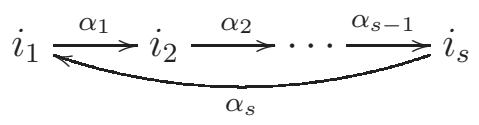

and $t=1,2, \cdots, s$, where $m=\operatorname{mult}(C)$ and the indices are considered in modulo $s$. (ii) $\alpha \beta$ if $\beta \neq \mathrm{na}(\alpha)$. 
(iii) $\left(\alpha_{1} \alpha_{2} \cdots \alpha_{s}\right)^{m}-\left(\beta_{1} \beta_{2} \cdots \beta_{t}\right)^{m^{\prime}}$ whenever we have a diagram

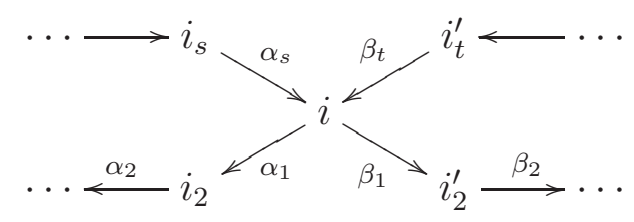

where $C_{\alpha_{\ell}}=C_{\alpha_{1}}, C_{\beta_{\ell^{\prime}}}=C_{\beta_{1}}$ for any $1 \leq \ell \leq s, 1 \leq \ell^{\prime} \leq t$ and $m=\operatorname{mult}\left(C_{\alpha_{1}}\right), m^{\prime}=$ $\operatorname{mult}\left(C_{\beta_{1}}\right)$.

We define a $k$-algebra $A:=A_{(Q, \mathrm{C})}$ associated with $(Q, \mathrm{C})$ by $A=k Q / I_{(Q, \mathrm{C})}$. Then the algebra $A_{(Q, \mathrm{C})}$ is finite dimensional and symmetric. The cycle-decomposition $\mathrm{C}$ is also said to be the cycle-decomposition of $A_{(Q, \mathrm{C})}$.

An algebra $A:=k Q / I$ is said to be special biserial if $Q$ is special and for any arrow $\beta$ of $Q$, there is at most one arrow $\alpha$ with $\alpha \beta \notin I$ and at most one arrow $\gamma$ with $\beta \gamma \notin I$.

Thanks to [Ro, An] (see Proposition 5.5), we have the following result.

Lemma 2.3. The assignment $(Q, \mathrm{C}) \mapsto A_{(Q, \mathrm{C})}$ gives rise to a bijection between the isoclasses of $S B$ quivers and those of symmetric special biserial algebras.

Example 2.4. (1) Let $Q$ be the quiver

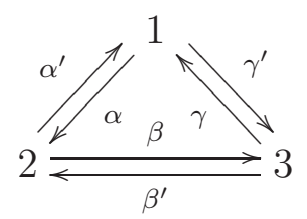

with the relations $I:=\left\langle\alpha \beta, \beta \gamma, \gamma \alpha, \alpha^{\prime} \gamma^{\prime}, \gamma^{\prime} \beta^{\prime}, \beta^{\prime} \alpha^{\prime}, \alpha^{\prime} \alpha-\beta \beta^{\prime}, \beta^{\prime} \beta-\gamma \gamma^{\prime}, \gamma^{\prime} \gamma-\alpha \alpha^{\prime}\right\rangle$. Then the algebra $A:=k Q / I$ is symmetric special biserial associated with the $\mathrm{SB}$ quiver $(Q, \mathrm{C})$ where the cycle-decomposition is

$$
\mathbf{C}=\left\{\left(1 \underset{\alpha^{\prime}}{\stackrel{\alpha}{\rightleftarrows}} 2\right),\left(2 \underset{\beta^{\prime}}{\stackrel{\beta}{\rightleftarrows}} 3\right),\left(3 \underset{\gamma^{\prime}}{\stackrel{\gamma}{\rightleftarrows}} 1\right)\right\}
$$

such that the multiplicity of every cycle is 1 .

(2) Let $Q$ be the quiver

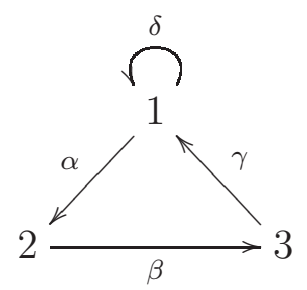

with the relations $I:=\left\langle\gamma \alpha,(a b c d)^{2} a \mid\{a, b, c, d\}=\{\alpha, \beta, \gamma, \delta\}\right\rangle$. Then $A:=k Q / I$ is a symmetric special biserial algebra which is isomorphic to $A_{(Q, \mathrm{C})}$, where $\mathrm{C}$ is the cycle-decomposition

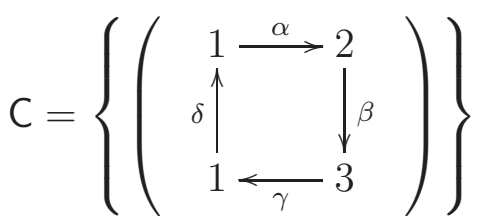

with the multiplicity 2 . 
(3) Let $Q$ be the quiver

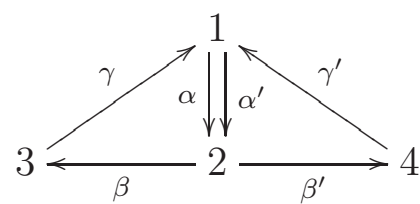

with cycle-decomposition $\mathrm{C}=\left\{C_{1}, C_{2}\right\}$ where

$$
C_{1}=\left(\begin{array}{c}
\gamma \nearrow^{1} \downarrow_{\alpha} \\
3 \underset{\beta}{\stackrel{\gamma}{<}}
\end{array}\right), C_{2}=\left(\begin{array}{r}
1 \\
\alpha^{\prime} \downarrow \\
2 \underset{\beta^{\prime}}{\longrightarrow} 4
\end{array}\right)
$$

and $\operatorname{mult}\left(C_{1}\right)=\operatorname{mult}\left(C_{2}\right)=1$. Then we have an isomorphism $A_{(Q, \mathrm{C})} \simeq k Q / I$ where $I=\left\langle\alpha \beta^{\prime}, \alpha^{\prime} \beta, \gamma \alpha^{\prime}, \gamma^{\prime} \alpha,(a b c) a \mid\{a, b, c\}=\{\alpha, \beta, \gamma\},\left\{\alpha^{\prime}, \beta^{\prime}, \gamma^{\prime}\right\}\right\rangle$.

We know that the property of being symmetric special biserial is derived invariant.

Proposition 2.5. Let $A$ and $B$ be finite dimensional algebras. Suppose that $A$ and $B$ are derived equivalent. If $A$ is a symmetric special biserial algebra, then so is $B$.

Proof. Combine [R1] and [P].

Next, we recall the notion of tilting mutation. We refer to [AI for details.

The bounded derived category of $\bmod A$ is denoted by $\mathrm{D}^{\mathrm{b}}(\bmod A)$.

We give the definition of tilting complexes.

Definition 2.6. Let $A$ be a finite dimensional algebra. We say that a bounded complex $T$ of finitely generated projective $A$-modules is tilting if it satisfies $\operatorname{Hom}_{\mathrm{D}^{\mathrm{b}}(\bmod A)}(T, T[n])=0$ for any integer $n \neq 0$ and produces the complex $A$ concerned in degree 0 by taking direct summands, mapping cones and shifts.

The following result shows the importance of tilting complexes.

Theorem 2.7. [R1] Let $A$ and $B$ be finite dimensional algebras. Then $A$ and $B$ are derived equivalent if and only if there exists a tilting complex $T$ of $A$ such that $B$ is Morita equivalent to the endomorphism algebra $\operatorname{End}_{\mathrm{D}^{\mathrm{b}}(\bmod A)}(T)$.

For each vertex $i$ of $Q$, we denote by $e_{i}$ the corresponding primitive idempotent of $A$.

We recall a complex given by Okuyama and Rickard [O, R2], which is a special case of tilting mutation (see $[\mathrm{AI}]$ ).

Definition-Theorem 2.8. O] Fix a vertex $i$ of $Q$. We define a complex by

$$
T_{j}:=\left\{\begin{array}{cc}
(0 \text { th }) & (1 \mathrm{st}) \\
P_{j} \longrightarrow 0 & (j \neq i) \\
P \longrightarrow \pi_{i} \longrightarrow P_{i} & (j=i)
\end{array}\right.
$$

where $P \stackrel{\pi_{i}}{\longrightarrow} P_{i}$ is a minimal projective presentation of $e_{i} A / e_{i} A\left(1-e_{i}\right) A$. Now we call $T(i):=\bigoplus_{j \in Q_{0}} T_{j}$ an Okuyama-Rickard complex with respect to $i$ and put $\mu_{i}^{+}(A):=$ $\operatorname{End}_{\mathrm{D}^{\mathrm{b}}(\bmod A)}(T(i))$. If $A$ is symmetric, then $T$ is tilting. In particular, $\mu_{i}^{+}(A)$ is derived equivalent to $A$. 


\section{Mutation of SB quivers}

The aim of this paper is to give a purely combinatorial description of tilting mutation of symmetric special biserial algebras.

To do this, we introduce mutation of $S B$ quivers by dividing to three cases, which is a new SB quiver $\mu_{i}^{+}(Q, \mathrm{C})$ made from a given one $(Q, \mathrm{C})$.

Now, the main theorem in this paper is stated, which gives the compatibility between tilting mutation and mutation of SB quivers. This is proved in Section 6 .

Theorem 3.1. Let $A$ be a symmetric special biserial algebra and take a $S B$ quiver $(Q, C)$ satisfying $A \simeq A_{(Q, \mathrm{C})}$. Let $i$ be a vertex of $Q$. Then we have an isomorphism $A_{\mu_{i}^{+}(Q, \mathrm{C})} \simeq$ $\mu_{i}^{+}(A)$. In particular, $A_{\mu_{i}^{+}(Q, \mathrm{C})}$ is derived equivalent to $A$.

Let $(Q, \mathrm{C})$ be a SB quiver and $i$ be a vertex of $Q$. We say that $Q$ is multiplex at $i$ if there exists arrows $i \underset{\beta}{\stackrel{\alpha}{\rightleftarrows}} j$ with $\beta \neq \mathrm{na}(\alpha)$ and $\alpha \neq \mathrm{na}(\beta)$.

3.1. Non-multiplex case. We introduce mutation of SB quivers at non-multiplex vertices.

Let $(Q, \mathrm{C})$ be a SB quiver and fix a vertex $i$ of $Q$. We define a new SB quiver $\mu_{i}^{+}(Q, \mathrm{C})=$ $\left(Q^{\prime}, C^{\prime}\right)$ as follows.

\subsubsection{Mutation rules.}

Definition 3.2. Suppose that $Q$ is non-multiplex at $i$. We define a quiver $Q^{\prime}$ as the following three steps:

(QM1) Consider any path

$$
h \stackrel{\alpha}{\longrightarrow} i \stackrel{\beta}{\longrightarrow} j \text { with } \beta=\mathrm{na}(\alpha) \quad \text { or } \quad h \stackrel{\alpha}{\longrightarrow} i \stackrel{\beta}{\longrightarrow} j \text { with } \gamma=\mathrm{na}(\alpha), \beta=\mathrm{na}(\gamma)
$$

for $h \neq i \neq j$. Then draw a new arrow $h \stackrel{x}{\longrightarrow} j$

(QM1-1) if $h \neq j$ or

$(\mathrm{QM} 1-2)$ if $h=j, \alpha=\operatorname{na}(\beta)$ and $\operatorname{mult}\left(C_{\alpha}\right)>1$.

(QM2) Remove all arrows $h \longrightarrow i$ for $h \neq i$.

(QM3) Consider any arrow $i \stackrel{\alpha}{\longrightarrow} h$ for $h \neq i$.

(QM3-1) If there exists a path $i \stackrel{\alpha}{\longrightarrow} h \stackrel{\beta}{\longrightarrow} j$ with $\beta \neq$ na $(\alpha)$, then replace it by a new path $h \stackrel{x}{\longrightarrow} i \stackrel{y}{\longrightarrow} j$.

(QM3-2) Otherwise, add a new arrow $h \stackrel{x}{\longrightarrow} i$.

It is easy to see that the new quiver $Q^{\prime}$ is again special.

3.1.2. Cycle-decompositions. We give a cycle-decomposition $C^{\prime}$ of $Q^{\prime}$.

Definition 3.3. We use the notation of Definition 3.2 .

(1) We define a cycle containing a new arrow $x$ in (QM1) as follows:

(i) In the case (QM1-1), $C_{x}$ is obtained by replacing $\alpha \beta$ or $\alpha \gamma \beta$ in $C_{\alpha}$ by $x$.

(ii) In the case $(\mathrm{QM1}-2), C_{x}$ is a new cycle $h \supset x$ with multiplicity $\operatorname{mult}\left(C_{\alpha}\right)$. 
(2) We define a cycle containing a new arrow $x$ and $y$ in (QM3) as follows:

(i) In the case (QM3-1), $C_{x}=C_{y}$ and replace $\beta$ in $C_{\beta}$ by $x y$.

(ii) In the case (QM3-2),

(a) if there exists an arrow $h \stackrel{\beta}{\longrightarrow} i$ of $Q$, then $C_{x}$ is defined by replacing $\beta$ in $C_{\beta}$ by $x$.

(b) Otherwise, $C_{x}$ is a new cycle

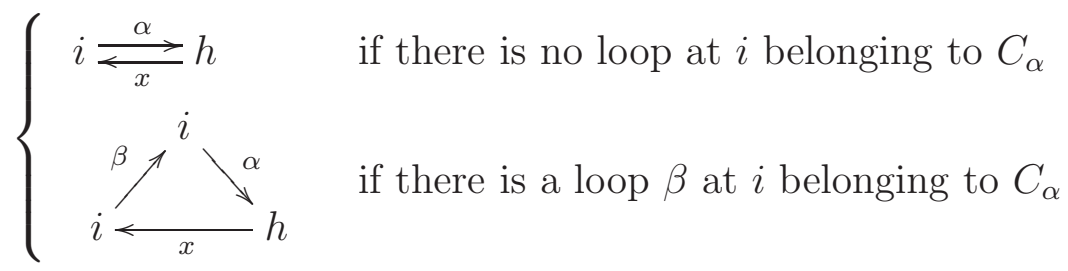

with multiplicity 1.

Then we obtain a cycle-decomposition $\mathrm{C}^{\prime}$ of $Q^{\prime}$.

Thus, we get a new SB quiver $\mu_{i}^{+}(Q, \mathrm{C}):=\left(Q^{\prime}, \mathrm{C}^{\prime}\right)$, called right mutation of $(Q, \mathrm{C})$ at $i$.

Dually, we define the left mutation $\mu_{i}^{-}(Q, \mathrm{C})$ of $(Q, \mathrm{C})$ at $i$ by $\mu_{i}^{-}(Q, \mathrm{C}):=$ $\mu_{i}^{+}\left(Q^{\mathrm{op}}, \mathrm{C}^{\mathrm{op}}\right)^{\mathrm{op}}$, where $Q^{\mathrm{op}}$ is the opposite quiver of $Q$ and $\mathrm{C}^{\mathrm{op}}$ is the cycle-decomposition of $Q^{\text {op }}$ corresponding to C.

Example 3.4. (1) Let $(Q, \mathrm{C})$ be the SB quiver as in Example 2.4 (1). Then we have the right mutation $\mu_{1}^{+}(Q, \mathrm{C})=\left(Q^{\prime}, \mathrm{C}^{\prime}\right)$ of $Q$ at 1 as follows:

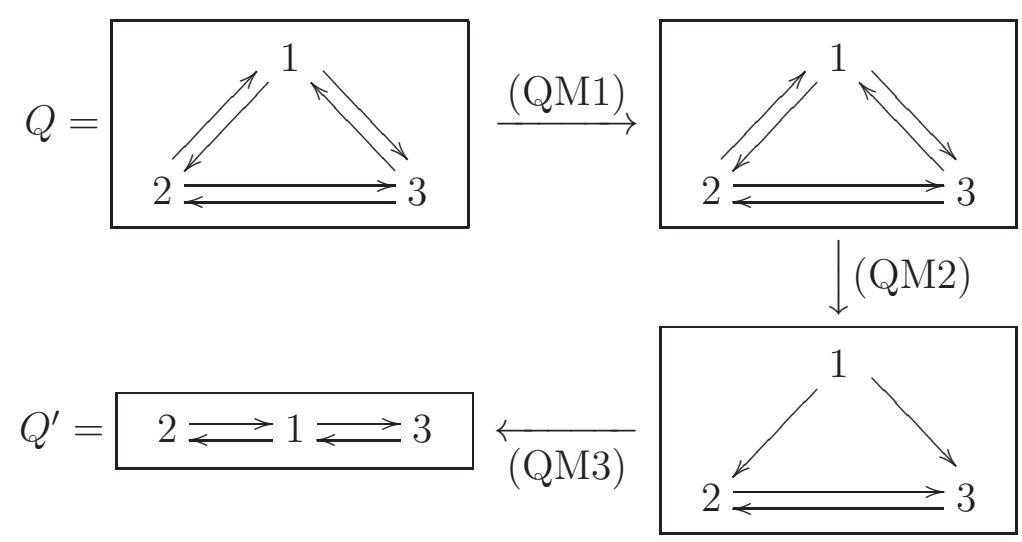

and

$$
C^{\prime}=\left\{\left(\begin{array}{ll}
1 \longrightarrow 2 \\
\uparrow & \\
& \downarrow \\
3 \longleftrightarrow & 1
\end{array}\right)\right\}
$$


(2) Let $(Q, \mathrm{C})$ be the SB quiver of Example 2.4 (2). Then the right mutation $\mu_{1}^{+}(Q, \mathrm{C})=$ $\left(Q^{\prime}, C^{\prime}\right)$ of $Q$ at 1 is obtained as follows:
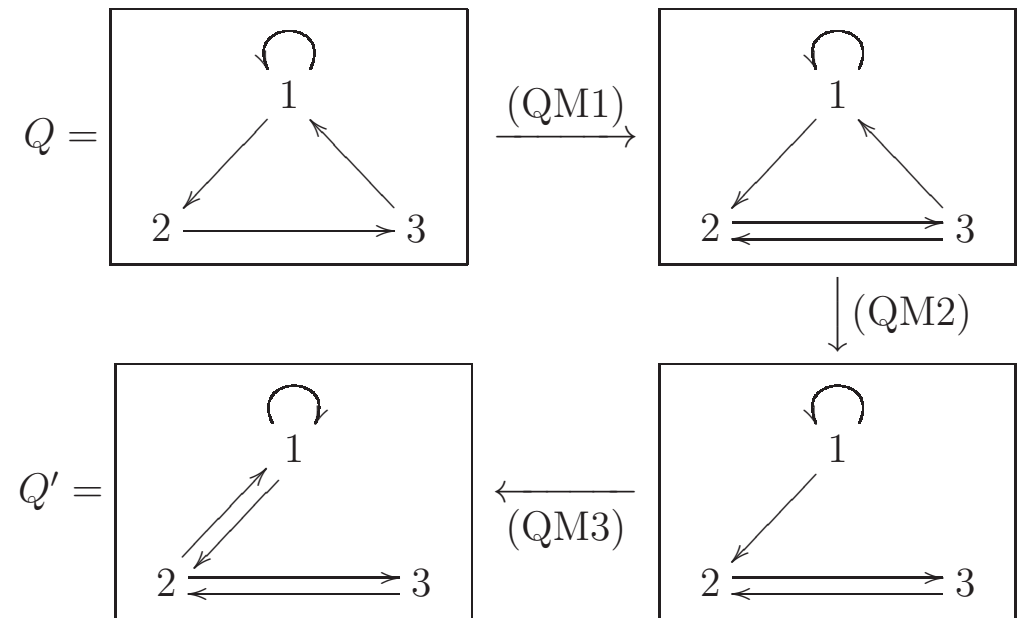

and

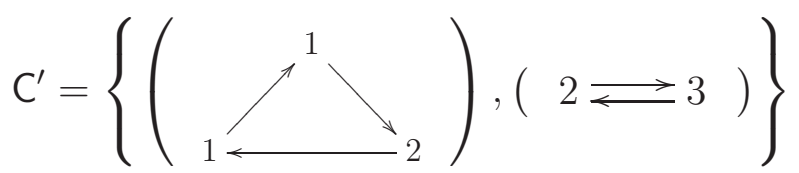

where the first and the second cycles have multiplicity 1 and 2, respectively.

(3) Let $(Q, \mathrm{C})$ be the SB quiver as in Example 2.4 (3). Then we get the right mutation $\mu_{1}^{+}(Q, \mathrm{C})=\left(Q^{\prime}, \mathrm{C}^{\prime}\right)$ of $Q$ at 1 as follows:
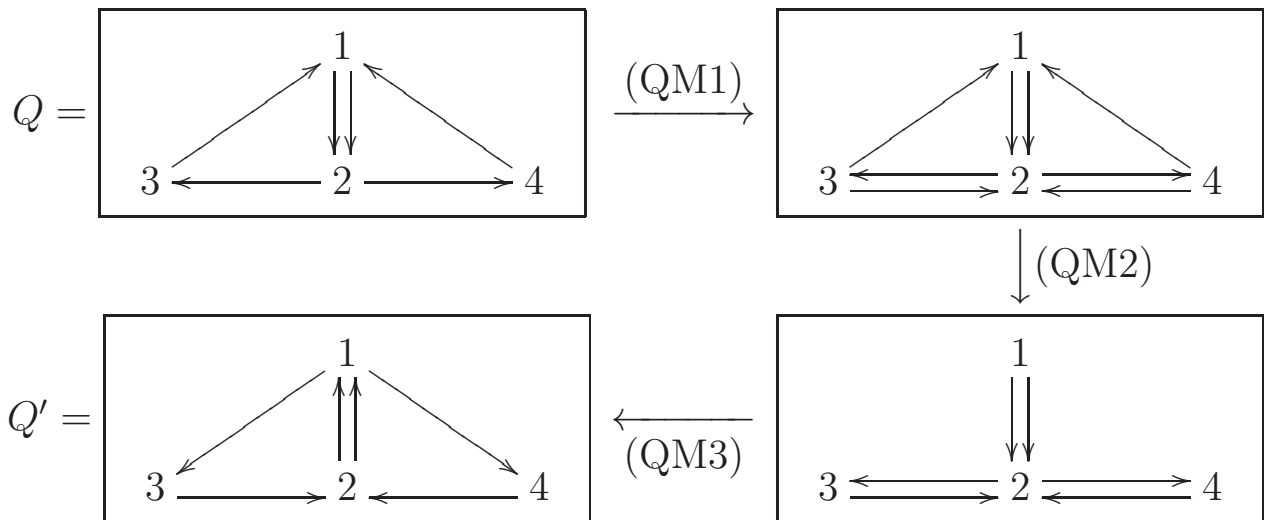

and

$$
C^{\prime}=\left\{\left(\begin{array}{l}
1 \\
\uparrow \\
3 \stackrel{\swarrow}{\longrightarrow}
\end{array}\right),\left(\begin{array}{l}
1 \\
\uparrow \searrow \\
2 \longleftarrow 4
\end{array}\right)\right\}
$$

3.2. Multiplex case (1). Next, we introduce mutation at multiplex vertices and its cycle-decomposition. They are defined by making minor alterations to mutation at nonmultiplex vertices.

Let $(Q, \mathrm{C})$ be a SB quiver and fix a vertex $i$ of $Q$. We consider the following situation:

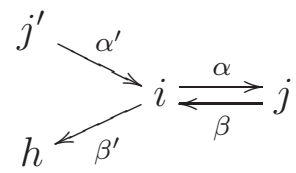


with $\beta \neq \mathrm{na}(\alpha)$ and $\alpha \neq \mathrm{na}(\beta)$ : in this case, it is observed that $\alpha=\mathrm{na}\left(\alpha^{\prime}\right)$ and $\beta^{\prime}=\mathrm{na}(\beta)$.

We define a new SB quiver $\mu_{i}^{+}(Q, \mathrm{C})=\left(Q^{\prime}, \mathrm{C}^{\prime}\right)$ as follows.

\subsubsection{Mutation rules.}

Definition 3.5. We assume that $j^{\prime} \neq h$. A quiver $Q^{\prime}$ of $Q$ at $i$ is defined by the following three steps:

(QM1)' Draw a new arrow $j^{\prime} \stackrel{x}{\longrightarrow} j$

(QM1-1)' if $j^{\prime} \neq j$ or

$(\mathrm{QM} 1-2)^{\prime}$ if $j^{\prime}=j$ and $\operatorname{mult}\left(C_{\alpha}\right)>1$.

(QM2)' Remove two arrows $\alpha$ and $\alpha^{\prime}$.

(QM3)' Add new arrows in the following way:

(QM3-1)' If there is an arrow $\gamma: h \longrightarrow h^{\prime}$ with $\gamma \neq$ na $\left(\beta^{\prime}\right)$, then remove $\gamma$ and add new arrows $h \stackrel{x}{\longrightarrow} i \stackrel{y}{\longrightarrow} h^{\prime}$.

(QM3-2)' Otherwise, add new arrows $h \underset{y}{\stackrel{x}{\rightleftarrows}} i$.

We can easily check that the new quiver $Q^{\prime}$ is again special.

3.2.2. Cycle-decompositions. We give a cycle-decomposition $C^{\prime}$ of $Q^{\prime}$.

Definition 3.6. Assume that $j^{\prime} \neq h$. We use the notation of Definition 3.5.

(1) We define a cycle containing a new arrow $x$ in (QM1)' as follows:

(i) In the case (QM1-1)', $C_{x}$ is obtained by replacing $\alpha^{\prime} \alpha$ in $C_{\alpha}$ by $x$.

(ii) In the case (QM1-2)', $C_{x}$ is a new cycle $j \bigcirc x$ with multiplicity $\operatorname{mult}\left(C_{\alpha}\right)$.

(2) We define a cycle containing a new arrow $x$ and $y$ in (QM3)' as follows:

(i) In the case (QM3-1)', $C_{x}=C_{y}$ and replace $\gamma$ in $C_{\gamma}$ by $x y$.

(ii) In the case (QM3-2)', $C_{x}$ and $C_{y}$ are new cycles satisfying $C_{x}=C_{y}=$ $(h \underset{y}{\stackrel{x}{\rightleftarrows}} i)$ with multiplicity 1 .

Then we have a cycle-decomposition $C^{\prime}$ of $Q^{\prime}$.

Thus, we get a new SB quiver $\mu_{i}^{+}(Q, \mathrm{C})=\left(Q^{\prime}, \mathrm{C}^{\prime}\right)$, called right mutation of $(Q, \mathrm{C})$ at $i$.

Dually, we define the left mutation $\mu_{i}^{-}(Q, \mathrm{C})$ of $(Q, \mathrm{C})$ at $i$ by $\mu_{i}^{-}(Q, \mathrm{C}):=$ $\mu_{i}^{+}\left(Q^{\mathrm{op}}, \mathrm{C}^{\mathrm{op}}\right)^{\mathrm{op}}$.

Example 3.7. Let $Q$ be the quiver

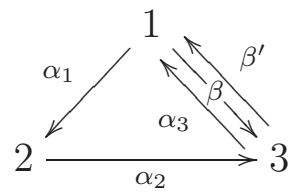

with cycle-decomposition C:

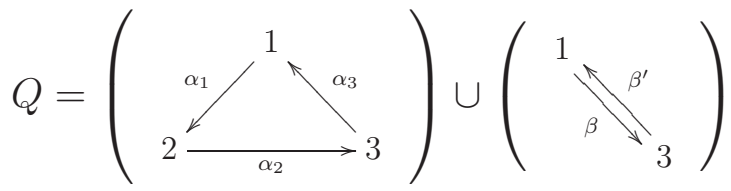


such that the multiplicity of each cycle is 1 . Then we see that the right mutation $\mu_{1}^{+}(Q, \mathrm{C})=\left(Q^{\prime}, \mathrm{C}^{\prime}\right)$ of $Q$ at 1 is

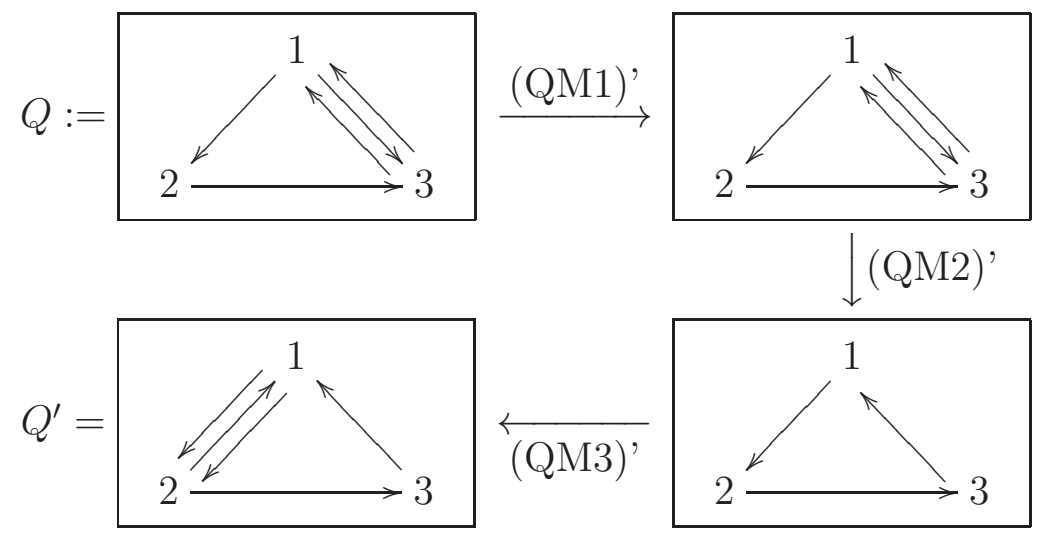

and

$$
C^{\prime}=\left\{\left(\swarrow_{2}^{1} \bigsqcup_{3}\right),\left(\mu^{1}\right)\right\}
$$

3.3. Multiplex case (2). Finally, we introduce the last case of mutation of SB quivers.

Let $(Q, \mathrm{C})$ be a SB quiver and fix a vertex $i$ of $Q$. Suppose that the subquiver of $Q$ around the vertex $i$ is

$$
j^{\prime} \underset{\beta^{\prime}}{\stackrel{\alpha^{\prime}}{\rightleftarrows}} i \underset{\beta}{\stackrel{\alpha}{\rightleftarrows}} j
$$

with $\beta \neq \mathrm{na}(\alpha)$ and $\alpha \neq \mathrm{na}(\beta)$ : i.e., the case of $j^{\prime}=h$ in Multiplex case $(1)$.

Definition 3.8. We define the right mutation $\mu_{i}^{+}(Q, \mathrm{C})$ of $(Q, \mathrm{C})$ at $i$ by $\mu_{i}^{+}(Q, \mathrm{C})=$ $(Q, \mathrm{C})$.

Dually, the left mutation $\mu_{i}^{-}(Q, \mathrm{C})$ of $(Q, \mathrm{C})$ at $i$ is also defined by $\mu_{i}^{-}(Q, \mathrm{C})=(Q, \mathrm{C})$.

\section{Reduction THEOREM}

The aim of this section is to give 'reduction' theorem for symmetric special biserial algebras, which is a generalization of Rickard's star theorem (see Section 5.3).

Throughout this section, let $A$ be a symmetric special biserial algebra associated with a $\mathrm{SB}$ quiver $(Q, \mathrm{C})$.

Note first that every $\mu_{i}^{+}$preserves the property of being (non-) multiplex, that is, $A$ is non-multiplex at any vertex of $Q$ if and only if so is $\mu_{i}^{+}(A)$.

The main theorem of this section is the following.

Theorem 4.1. Let $A$ be a symmetric special biserial algebra associated with a $S B$ quiver $\left(Q,\left\{C_{0}, C_{1}, \cdots, C_{s}\right\}\right)$ satisfying mult $\left(C_{0}\right) \geq \operatorname{mult}\left(C_{1}\right) \geq \cdots \geq \operatorname{mult}\left(C_{s}\right)$. Then the algebra $A$ is derived equivalent to a symmetric special biserial algebra with a cycle-decomposition $\left\{C_{0}^{\prime}, C_{1}^{\prime}, \cdots, C_{v}^{\prime}\right\}$ satisfying the following properties:

(1) All vertices appear in $C_{0}^{\prime}$;

(2) $v \leq 1$ if $\operatorname{mult}\left(C_{2}\right)=1$. Otherwise, $v=\max \left\{\ell \in\{2, \cdots, s\} \mid \operatorname{mult}\left(C_{\ell}\right) \neq 1\right\}$;

(3) $\operatorname{mult}\left(C_{\ell}^{\prime}\right)=\operatorname{mult}\left(C_{\ell}\right)$ for any $0 \leq \ell \leq v$;

(4) Each $C_{\ell}^{\prime}$ for $\ell \geq 2$ is a loop. 
To show this, we introduce a method for reducing some cycles.

Method 4.2. Let $(Q, \mathrm{C})$ be a SB quiver and $C_{0}$ a cycle of $Q$. We construct a new SB quiver $\left(Q^{\prime}, C^{\prime}\right)$ as follows.

(R1) Let $i$ be a vertex of $Q$ which does not belong to $C_{0}$ and assume that there exist arrows $i \stackrel{\alpha}{\longrightarrow} h \stackrel{\beta}{\longrightarrow} j$ with $C_{0}=C_{\beta}$. Then mutating at $i$, we obtain new arrows $h \stackrel{x}{\longrightarrow} i \stackrel{y}{\longrightarrow} j$ of $\mu_{i}^{+}(Q, \mathrm{C})$ with $C_{x}=C_{y}$. Thus, we observe that the vertex $i$ belongs to $C_{x}$ : Again, write $C_{x}$ by $C_{0}$.

Repeating this argument, we get a SB quiver $\left(Q^{\prime}, C^{\prime}\right)$ having a cycle to which all vertices belong.

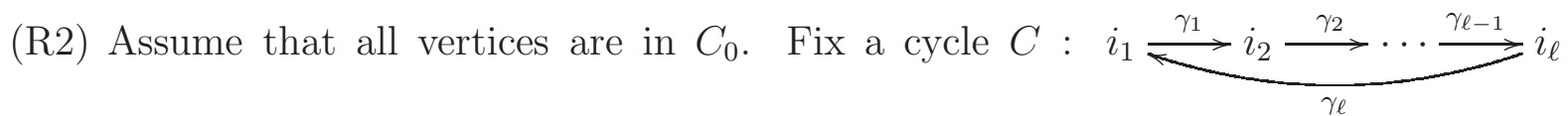
$\left(i_{1} \neq i_{2}\right)$ of $Q$ which is not $C_{0}$. Let $\alpha: i_{1} \longrightarrow h$ be an arrow in $C_{0}$. Suppose that $P_{h}$ is uniserial or that there exists an arrow $h \stackrel{\beta}{\longrightarrow} h^{\prime}$ with $C_{\beta} \neq C_{0}$ and $h \neq i_{\ell}$. We consider the following situation

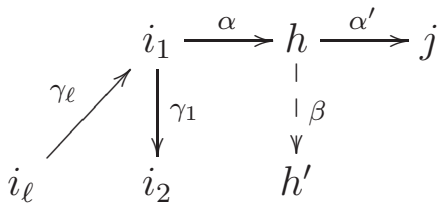

where $C_{\alpha}=C_{\alpha^{\prime}}=C_{0}$. Then mutating at $i_{1}$, we see that the vertex $i_{1}$ is in a new cycle $i_{1} \stackrel{\alpha}{\rightleftarrows} h$ or in $C_{\beta}$, and does not belong to $C_{\gamma_{2}}$. Since the vertex $i_{2}$ is contained in $C_{0}$, the vertex $i_{1}$ belongs to the cycle $C_{\alpha^{\prime}}$ of $\mu_{i}^{+}(Q)$ as well as all the other vertices: Again, write $C_{\alpha^{\prime}}$ by $C_{0}$.

Continuing this argument, we get a SB quiver $\left(Q^{\prime}, C^{\prime}\right)$ satisfying the following:

(i) $\mathrm{C}^{\prime}$ admits a cycle $C_{0}$ to which all vertices belong.

(ii) The vertex $i_{\ell}$ is only in $C_{0}$ if $\operatorname{mult}(C)=1$, otherwise there is a loop at $i_{\ell}$ with multiplicity mult $(C)$.

(iii) $C^{\prime}$ has a cycle of the form

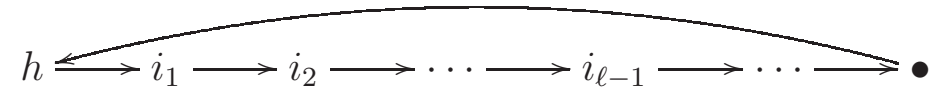

with multiplicity 1 or $\operatorname{mult}\left(C_{\beta}\right)$.

(R3) Assume that all vertices are in $C_{0}$. Let $i$ be a vertex of $Q$ having a loop $i \supset \alpha$ with $C_{\alpha} \neq C_{0}$. Let $h \longrightarrow i \longrightarrow j$ be a path in $C_{0}$. Then mutating at $i$ twice, we obtain a SB quiver $\left(Q^{\prime}, C^{\prime}\right)$ satisfying the following:

(i) $C^{\prime}$ admits a cycle $C_{0}$ to which all vertices belong.

(ii) There is an arrow $h \longrightarrow j$ in $C_{0}$.

(iii) There is a loop $\alpha^{\prime}$ at $i$ with $C_{\alpha^{\prime}} \neq C_{0}$.

Now we are ready to prove Theorem 4.1.

Proof of Theorem 4.1. By applying (R1), we can assume that all vertices are in $C_{0}$. Finish if $s \leq 1$. 
Assume $s \geq 2$ and let $C_{s}$ be a cycle of the form $i_{1} \longrightarrow i_{2} \longrightarrow \cdots \longrightarrow i_{\ell}$.

(i) If $C_{s}$ is not a loop, then we do (R2) for $C_{s}$ : Denote by $\left(Q^{\prime}, \mathrm{C}^{\prime}\right)$ the new $\mathrm{SB}$ quiver. There exists a cycle $C_{0}^{\prime}$ in $C^{\prime}$ to which all vertices belong and it is observed that the vertex $i_{\ell}$ of $Q^{\prime}$ is only in $C_{0}^{\prime}$ or there is a loop at $i_{\ell}$. Moreover, there exists a cycle $C$ in $C^{\prime}$ having of the form $h \rightleftarrows i_{1} \longrightarrow \cdots \longrightarrow i_{\ell-1} \longrightarrow \cdots \longrightarrow \bullet$. If the number of vertices in $C$ is greater than $\ell$, then we obtain that the number of cycles in $C^{\prime}$ is at most $s$.

Assume that $C$ is just of the form $h \rightleftharpoons i_{1} \longrightarrow \cdots \longrightarrow i_{\ell-1}$. Let $i$ be the vertex of $Q^{\prime}$ having the unique arrow $h \rightarrow i$ in $C_{0}^{\prime}$. By applying (R3) if necessary, we may suppose that there is no loop at $i$. As $s \geq 2$, we can also assume that there is an arrow $i \stackrel{\beta}{\rightarrow} j$ of $Q^{\prime}$ with $C_{\beta} \neq C_{0}^{\prime}$. Doing (R2) for $C$, one gets a new SB quiver $\left(Q^{\prime \prime},\left\{C_{0}^{\prime \prime}, \cdots, C_{v}^{\prime \prime}\right\}\right)$ with $v \leq s$.

(ii) Let $t \geq 2$. Assume that $C_{t+1}, \cdots, C_{s}$ are loops but $C_{t}$ is not a loop. Put $C_{t}:=\left\{i_{1} \longrightarrow i_{2} \longrightarrow \cdots \longrightarrow i_{\ell}\right\}$ and let $h$ be the vertex having the unique arrow $i_{1} \rightarrow h$ in $C_{0}$. By applying (R3) if necessary, we may suppose that $h$ does not belong to $C_{t^{\prime}}$ for $t+1 \leq t^{\prime} \leq s$. Then do (R2) for $C_{t}$ and let $\left(Q^{\prime}, \mathrm{C}^{\prime}\right)$ be the new $\mathrm{SB}$ quiver. We see that there is a loop at $i_{\ell}$ in $Q^{\prime}$ and $C^{\prime}$ has a cycle $C$ of the form $h \rightleftharpoons i_{1} \longrightarrow \cdots \longrightarrow i_{\ell-1} \longrightarrow \cdots \longrightarrow \bullet$. By the same argument in (i), we can get a SB quiver $\left(Q^{\prime \prime},\left\{C_{0}^{\prime \prime}, \cdots, C_{s}^{\prime \prime}\right\}\right)$ such that $C_{u}^{\prime \prime}$ is a loop for $t \leq u \leq s$.

Thus, the proof is complete.

\section{BRAUER GRAPH ALGEBRAS}

In this section, we introduce a flip of Brauer graphs and show that it is compatible with a tilting mutation of Brauer graph algebras. In particular any Brauer graph algebra is symmetric special biserial (and converse also holds [Ro]), therefore all the theorems stated in the previous sections are applied to Brauer graph algebras.

We recall the definition of Brauer graphs.

Definition 5.1. A Brauer graph $G$ is a graph with the following data:

(i) There exists a cyclic ordering of the edges adjacent to each vertex, usually described by the clockwise ordering.

(ii) For every vertex $v$, there exists a positive integer $m_{v}$ assigned to $v$, called the $m u l$ tiplicity of $v$. We say that a vertex $v$ is exceptional if $m_{v}>1$

5.1. Flip of Brauer graphs. Let $G$ be a Brauer graph. For a cyclic ordering $(\cdots, i, j, \cdots)$ adjacent to a vertex $v$ with $j \neq i$, we write $j$ by $e_{v}(i)$ and denote by $v_{j}(i)$ (simply, $v(i)$ ) the vertex of $j$ distinct from $v$ if it exists, otherwise $v(i):=v$.

We say that an edge $i$ of $G$ is external if it has a vertex with cyclic ordering which consists of only $i$, otherwise it is said to be internal.

We now introduce flip of Brauer graphs.

Definition 5.2. Let $G$ be a Brauer graph and fix an edge $i$ of $G$. We define the flip $\mu_{i}^{+}(G)$ of $G$ as follows:

Case (1) The edge $i$ has the distinct two vertices $v$ and $u$ : 
- If $i$ is internal, then

(Step 1) detach $i$ from $v$ and $u$;

(Step 2) attach it to $v(i)$ and $u(i)$ by $e_{v(i)}\left(e_{v}(i)\right)=i$ and $e_{u(i)}\left(e_{u}(i)\right)=i$, respectively.

Locally there are the following three cases:

(i)

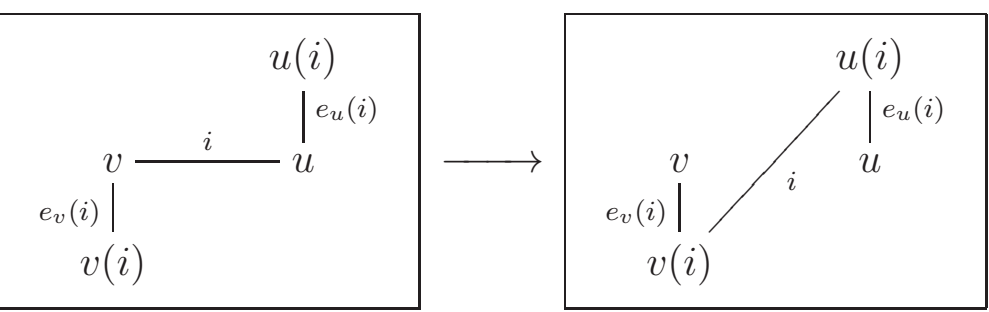

(ii)

(iii)

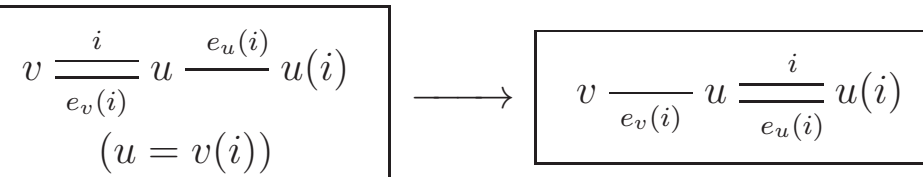

- If $i$ is external, namely $u$ is at end, then

$$
\begin{gathered}
0 \longrightarrow \frac{i}{\overline{e_{v}(i)}} u \\
\left(e_{u}(i)=e_{v}(i)\right)
\end{gathered} \quad \longrightarrow \quad v \frac{e_{v}(i)}{i} u
$$

(Step 1) detach $i$ from $v$;

(Step 2) attach it to $v(i)$ by $e_{v(i)}\left(e_{v}(i)\right)=i$.

The local picture is the following:

(iv)

Case (2) The edge $i$ has only one vertex $v$ :

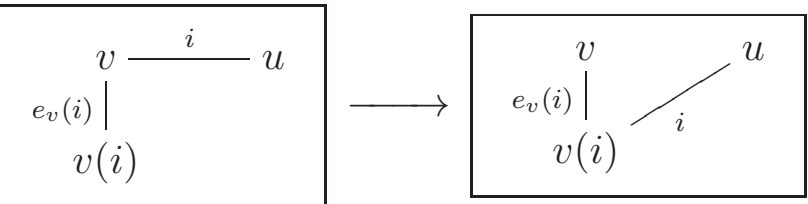

- If there exists the distinct two edges $h$ and $j$ written by $e_{v}(i)$, then (Step 1) detach $i$ from $v$;

(Step 2) attach it to $v_{h}(i)$ and $v_{j}(i)$ by $e_{v_{h}(i)}(h)=i$ and $e_{v_{j}(i)}(j)=i$.

Locally there are the following two cases:

(v) 

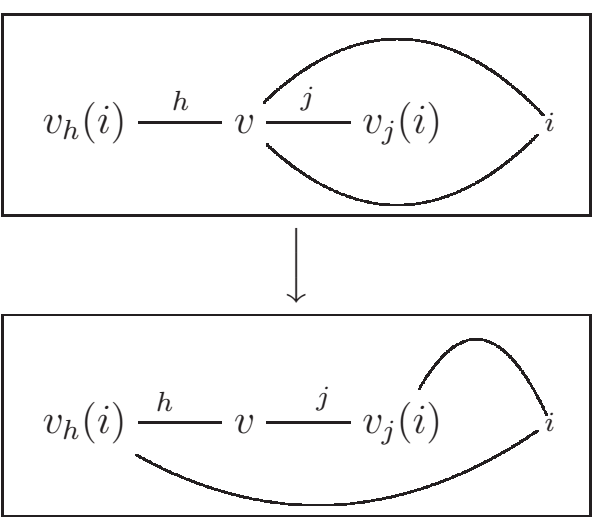

(vi)

- Otherwise,
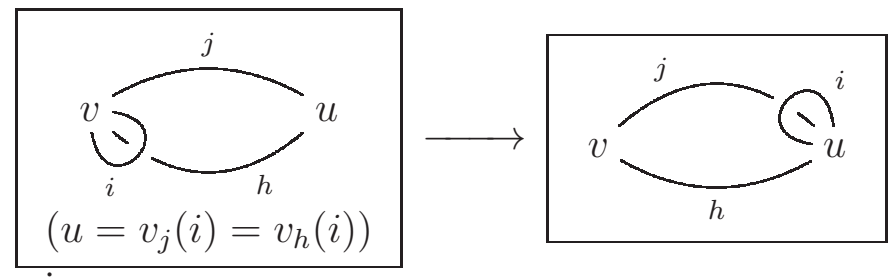

(Step 1) detach $i$ from $v$;

(Step 2) attach it to the only one vertex $v(i)$ by $e_{v(i)}\left(e_{v}(i)\right)=i$.

The local picture is the following:

(vii)

$$
\bigcap_{v \frac{i}{e_{v}(i)} v(i)} \longrightarrow \bigcap_{v}^{i}
$$

In all cases, the multiplicity of any vertex does not change.

Dually, we define $\mu_{i}^{-}(G)$ by $\mu_{i}^{-}(G):=\left(\mu_{i}^{+}\left(G^{\mathrm{op}}\right)\right)^{\text {op }}$ where the opposite Brauer graph, namely its cyclic ordering is described by counter-clockwise, is denoted by $G^{\text {op }}$.

Every case of flip of Brauer graphs is covered in Definition 5.2.

We also point out that our flip of Brauer graphs can be regarded as a generalization of flip of triangulations of surfaces [FST, MS].

Example 5.3. For a Brauer graph, we denote by $\bullet$ an exceptional vertex and by $\circ$ a non-exceptional vertex.

(1) Let $G$ be the Brauer graph

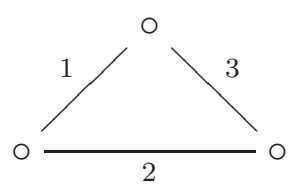

Then the flip of $G$ at 1 is

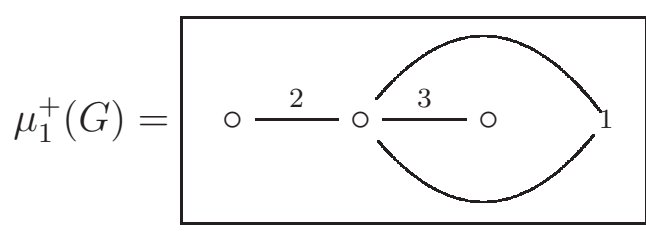


(2) Let $G$ be the Brauer graph

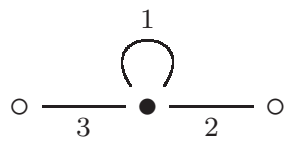

such that the multiplicity of the exceptional vertex $\bullet$ is 2 . Then we have the flip of $G$ at 1:

$$
\mu_{1}^{+}(G)=\overbrace{0}^{1}
$$

(3) Let $G$ be the Brauer graph

$$
\circ \frac{3}{\frac{0-4=}{2} \bigcirc}
$$

Then the flip of $G$ at 1 is observed by

$$
\mu_{1}^{+}(G)=\circ \frac{3}{\frac{0-4=}{1} \bigcirc}
$$

(4) Let $G$ be the Brauer graph

$$
\circ \frac{1}{3} \circ \frac{2}{\circ} \circ
$$

Then the flip of $G$ at 1 is:

$$
\mu_{1}^{+}(G)=\circ \frac{3}{2} \circ \frac{1}{2} \circ
$$

5.2. Compatibility of flip and tilting mutation. For a Brauer graph $G$, we denote by $\operatorname{vx}(G)$ the set of the vertices of $G$.

We construct a SB quiver from a Brauer graph.

Definition 5.4. Let $G$ be a Brauer graph. A Brauer quiver $Q=Q_{G}$ is a finite quiver given by a Brauer graph $G$ as follows:

(i) There exists a one-to-one correspondence between vertices of $Q$ and edges of $G$.

(ii) For two distinct edges $i$ and $j$, an arrow $i \longrightarrow j$ of $Q$ is drawn if there exists a cyclic ordering of the form $(\cdots, i, j, \cdots)$.

(iii) For an edge $i$ of $G$, we draw a loop at $i$ if it has an exceptional vertex which is at end.

Then $Q$ is special.

For each vertex $v$ of $G$, let $\left(i_{1}, i_{2}, \cdots, i_{s}, i_{1}\right)$ be a cyclic ordering at $v$. Then we define a cycle $C_{v}$ by

$$
i_{1} \longleftrightarrow i_{2} \longrightarrow \cdots \longrightarrow i_{s}
$$

with multiplicity $m_{v}$ if $s \neq 1$, otherwise by an empty set. We have a cycle-decomposition $\mathrm{C}=\mathrm{C}_{G}=\left\{C_{v} \mid v \in \operatorname{vx}(G)\right\}$.

Thus we obtain a SB quiver $(Q, \mathrm{C})$. 
For a Brauer graph $G$, a Brauer graph algebra $A=A_{G}$ is a symmetric special biserial algebra associated with the SB quiver $\left(Q_{G}, \mathrm{C}_{G}\right)$.

It is known that the notion of Brauer graph algebras is nothing but that of symmetric special biserial algebras. The following result is obtained.

Proposition 5.5. (1) [Ro, $\mathrm{An}]$ An algebra is a Brauer graph algebra if and only if it is symmetric special biserial.

(2) The property of being a Brauer graph algebra is derived invariant.

Proof. The second assertion follows from the first assertion and Proposition 2.5.

For an edge $i$ of a Brauer graph $G$, we say that $G$ has multi-edges at $i$ if there exists a subgraph $\stackrel{v}{\circ} \frac{i}{j} \stackrel{u}{\circ}$ of $G$ such that the cyclic orderings at $v$ and $u$ are $(\cdots, i, j, \cdots)$ and $(\cdots, j, i, \cdots)$, respectively; it is allowed that $u=v$.

We have the following easy observation.

Proposition 5.6. Let $G$ be a Brauer graph and $i$ be an edge of $G$. Then $Q_{G}$ is multiplex at $i$ if and only if $G$ has multi-edges at $i$.

It is not difficult to see that flip of each Brauer graph $G$ coincides with right mutation of the corresponding SB quiver $\left(Q_{G}, \mathrm{C}_{G}\right)$, that is, we have:

Proposition 5.7. Let $G$ be a Brauer graph and $i$ be an edge of $G$. Then one has $\left(Q_{\mu_{i}^{+}(G)}, \mathrm{C}_{\mu_{i}^{+}(G)}\right)=\mu_{i}^{+}\left(Q_{G}, \mathrm{C}_{G}\right)$.

We observe that Example $3.4(1)-(3)$ and Example 3.7 coincide with Example 5.3 (1)(3) and (4), respectively.

Applying Theorem 3.1 to Brauer graph algebras, we figure out that flip of Brauer graph is compatible with tilting mutation of Brauer graph algebras.

Theorem 5.8. Let $G$ be a Brauer graph and $i$ be an edge of $G$.

(1) We have an isomorphism $A_{\mu_{i}^{+}(G)} \simeq \mu_{i}^{+}\left(A_{G}\right)$.

(2) The algebra $\mu_{i}^{+}\left(A_{G}\right)$ has the Brauer graph $\mu_{i}^{+}(G)$.

(3) The algebra $A_{\mu_{i}^{+}(G)}$ is derived equivalent to $A_{G}$.

Proof. We have isomorphisms

$$
A_{\mu_{i}^{+}(G)} \simeq A_{\left(Q_{\mu_{i}^{+}(G)}, \mathrm{C}_{\mu_{i}^{+}(G)}\right)} \stackrel{5.7}{\simeq} A_{\mu_{i}^{+}\left(Q_{G}, \mathrm{C}_{G}\right)} \stackrel{3.1}{\simeq} \mu_{i}^{+}\left(A_{\left(Q_{G}, \mathrm{C}_{G}\right)}\right) \simeq \mu_{i}^{+}\left(A_{G}\right) .
$$

The second and the last assertion follow immediately.

Remark 5.9. Special cases of this theorem were given in [K], where he considered the cases (i)(iv) and (vii) in Definition 5.2 .

5.3. (Double-) Star theorem. In this subsection, we apply Theorem 4.1 to Brauer graph algebras and finally obtain a generalization of Rickard's star theorem.

Let $G$ be a Brauer graph. We denote by $\mathrm{m}_{G}$ the sequence $\left(m_{v_{1}}, \cdots, m_{v_{\ell}}\right)$ of the multiplicities of all vertices satisfying $m_{v_{1}} \geq \cdots \geq m_{v_{\ell}}$.

For a Brauer graph algebra $A$, the Brauer graph of $A$ is denoted by $G_{A}$.

A Brauer graph $G$ is said to be double-star if there exist two vertices $v$ and $u$ of $G$ such that any edge is either of the following: 
- It is external having the vertex $v$ :

- It has both the vertices $v$ and $u$ :

- It has only the vertex $v$, that is, it is of the form $v$.

We call $v$ and $u$ center and vice-center, respectively.

We say that a Brauer double-star $G$ satisfies multiplicity condition if the multiplicities of the center and the vice-center are the first and the second greatest among them of all vertices of $G$, respectively.

As a consequence of Theorem 4.1, the following theorem is obtained.

Theorem 5.10. Any Brauer graph algebra $A$ is derived equivalent to a Brauer graph algebra with Brauer double-star $G$ satisfying multiplicity condition such that

(i) the number of the edges of $G$ coincides with that of $G_{A}$ and

(ii) $\mathrm{m}_{G}=\mathrm{m}_{G_{A}}$, in particular $G$ and $G_{A}$ have the same number of exceptional vertices.

Proof. Let $\Lambda$ be a symmetric special biserial algebra with a cycle-decomposition $\mathrm{C}:=$ $\left\{C_{0}^{\prime}, \cdots, C_{v}^{\prime}\right\}$ as in Theorem 4.1 and put $G:=G_{\Lambda}$.

We first show that $G$ is a Brauer double-star. As the condition (1) in Theorem 4.1, the cycle $C_{0}^{\prime}$ corresponds to the center of $G$. We see that the vice-center of $G$ is given by the cycle $C_{1}^{\prime}$. Considering also the condition (4) of Theorem 4.1, we have that $G$ is a Brauer double-star.

By the condition (3) of Theorem 4.1, it is obtained that $G$ satisfies multiplicity condition. Since $A$ and $\Lambda$ are derived equivalent, they have the same number of non-isomorphic simple modules, whence the condition (i) is satisfied. As the condition (2) and (3) of Theorem 4.1, it follows that $G$ satisfies the condition (ii).

We raise a question on classification of derived equivalence classes of Brauer graph algebras.

Question 5.11. For a given Brauer graph $G$, is there up to isomorphism and opposite isomorphism a unique Brauer double-star algebra satisfying the multiplicity condition and which is derived equivalent to the algebra $A_{G}$ ?

It is well-known that this question has a positive answer if $G$ is a tree as a graph. Such a Brauer graph is said to be a generalized Brauer tree. It is called Brauer tree if it has at most one exceptional vertex. A (generalized) Brauer star is a (generalized) Brauer tree and a Brauer double-star. Note that any edge of a generalized Brauer star is external and every vertex can be a vice-center.

From Theorem 5.10, we deduce star theorem for generalized Brauer tree algebras.

Corollary 5.12. R2, M

(1) Any generalized Brauer tree algebra $A$ is derived equivalent to a generalized Brauer star algebra $B$ with $\mathrm{m}_{G_{B}}=\mathrm{m}_{G_{A}}$ such that the multiplicity of the center is maximal.

(2) Derived equivalence classes of generalized Brauer tree algebras are determined by the number of the edges and the multiplicities of the vertices.

\section{A PROOF OF MAIN THEOREM}

In this section we prove Theorem 3.1

Our proof of this theorem consists of three steps: The first is to decide the shape of the quiver of the algebra $\mu_{i}^{+}(A)$. The second is to give relations of $\mu_{i}^{+}(A)$, which is done by only determining every cycle. The last is to furnish the multiplicities to each cycle. 
6.1. Preliminaries. We prepare two important tools for our proof of the main theorem.

To determine the structure of a finite dimensional algebra $A$, the data $\operatorname{dim} \operatorname{Hom}_{A}\left(P_{i}, P_{j}\right)$ for projective $A$-modules $P_{i}$ and $P_{j}$, namely the Cartan data of $A$, play an important role. The following proposition gives the Cartan data of a derived equivalent algebra to $A$.

Proposition 6.1. [H] Let $A$ be a finite dimensional algebra. Let $T$ and $U$ be bounded complexes of projective A-modules satisfying $\operatorname{Hom}_{\mathrm{D}^{\mathrm{b}}(\bmod A)}(T, U[n])=0$ for any integer $n \neq 0$. Then the following equality holds:

$$
\operatorname{dim} \operatorname{Hom}_{\mathrm{D}^{\mathrm{b}}(\bmod A)}(T, U)=\sum_{\ell, m}(-1)^{\ell+m} \operatorname{dim} \operatorname{Hom}_{A}\left(T^{\ell}, U^{m}\right)
$$

where the $n$-th term of a complex $V$ is denoted by $V^{n}$.

We denote by $\underline{\bmod } A$ the stable category of $\bmod A$. It is well-known that $\underline{\bmod } A$ is triangulated category if $A$ is self-injective. Moreover, a derived equivalence between two self-injective algebras yields a stable equivalence between themselves [R2].

For an Okuyama-Rickard complex $T(i)$ of a symmetric algebra $A$, we denote by $F_{i}$ : $\underline{\bmod } A \rightarrow \underline{\bmod } \mu_{i}^{+}(A)$ the stable equivalence between $A$ and $\mu_{i}^{+}(A)$ induced by $T(i)$.

The syzygy of any module is denoted by $\Omega$.

Then we can exactly describe a $\mu_{i}^{+}(A)$-module $F_{i}(X)$ for every $A$-module $X$.

Proposition 6.2. [O] (see also [A, Lemma 3.4]) Let $A=k Q / I$ be a symmetric algebra and fix a vertex $i$ of $Q$. Then the following gives a complete list of simple $\mu_{i}^{+}(A)$-modules:

(1) $F_{i}\left(\Omega S_{i}\right)$;

(2) $F_{i}\left(S_{j}\right)$ if there is no such arrow $i \rightarrow j$ of $Q$;

(3) $F_{i}\left(X_{j}\right)$ where $X_{j}$ is maximal among submodules $X$ of $P_{j}$ such that only $S_{i}$ appears in a composition factor of $X / S_{j}$, if there is an arrow $i \rightarrow j$ of $Q$.

6.2. Extensions among simple modules. The shape of the quiver of a given algebra is determined by the dimensions of extensions among simple modules. This subsection is devoted to giving them for the endomorphism algebra of an Okuyama-Rickard complex of a symmetric special biserial algebra.

Throughout this subsection, assume that $A=k Q / I$ is a symmetric special biserial algebra and fix a vertex $i$ of $Q$. We put $B:=\mu_{i}^{+}(A)$ and still denote by $S_{j}$ a simple $B$-module corresponding to $T_{j}$ in Definition-Theorem 2.8 .

To calculate the dimensions of extensions among simple $B$-modules, we use Proposition 6.2 and feel free to utilize four formulas

$$
\begin{aligned}
& \operatorname{Ext}_{A}^{1}(X, Y) \simeq \operatorname{Ext}_{B}^{1}\left(F_{i} X, F_{i} Y\right) ; \quad \operatorname{Ext}_{A}^{1}(X, Y) \simeq \underline{\operatorname{Hom}}_{A}(\Omega X, Y) ; \\
& \underline{\operatorname{Hom}}_{A}(X, S)=\operatorname{Hom}_{A}(X, S) ; \quad \underline{\operatorname{Hom}}_{A}(S, X)=\operatorname{Hom}_{A}(S, X)
\end{aligned}
$$

for any $A$-modules $X, Y$ and simple $A$-module $S$.

Let $j$ and $j^{\prime}$ be vertices of $Q$ distinct from $i$.

(1) (i) If there is neither arrows $i \longrightarrow j$ nor $i \longrightarrow j^{\prime}$, then we observe an isomor$\operatorname{phism} \operatorname{Ext}_{B}^{1}\left(S_{j}, S_{j^{\prime}}\right) \simeq \operatorname{Ext}_{A}^{1}\left(S_{j}, S_{j^{\prime}}\right)$. 
(ii) If there is no arrow $i \longrightarrow j$ but is an arrow $i \longrightarrow j^{\prime}$, then we obtain isomorphisms

$$
\begin{aligned}
\operatorname{Ext}_{B}^{1}\left(S_{j}, S_{j^{\prime}}\right) & \simeq \operatorname{Ext}_{A}^{1}\left(S_{j}, X_{j^{\prime}}\right) \\
& \simeq \underline{\operatorname{Hom}}_{A}\left(S_{j}, \Omega^{-1} X_{j^{\prime}}\right) \\
& =\operatorname{Hom}_{A}\left(S_{j}, \Omega^{-1} X_{j^{\prime}}\right) .
\end{aligned}
$$

This implies that $\operatorname{dim} \operatorname{Ext}_{B}^{1}\left(S_{j}, S_{j^{\prime}}\right)$ is given by

$$
\begin{cases}1 & \text { if there is an arrow } j \longrightarrow j^{\prime} \text { of } Q ; \\ 1 & \text { if there is a cycle containing } j \longrightarrow i \longrightarrow j^{\prime} \text { of } Q ; \\ 1 & \text { if there is a cycle containing } j \longrightarrow i \longrightarrow j^{\prime} \text { of } Q ; \\ 2 & \text { if there are two cycles containing } j \longrightarrow j^{\prime} \text { of } Q ; \\ 0 & \text { otherwise. }\end{cases}
$$

(iii) If there is an arrow $i \longrightarrow j$ but is no arrow $i \longrightarrow j^{\prime}$, then we see isomorphisms

$$
\begin{aligned}
\operatorname{Ext}_{B}^{1}\left(S_{j}, S_{j^{\prime}}\right) & \simeq \operatorname{Ext}_{A}^{1}\left(X_{j}, S_{j^{\prime}}\right) \\
& \simeq \underline{\operatorname{Hom}}_{A}\left(\Omega X_{j}, S_{j^{\prime}}\right) \\
& =\operatorname{Hom}_{A}\left(\Omega X_{j}, S_{j^{\prime}}\right) .
\end{aligned}
$$

Thus we get that $\operatorname{dim}_{\operatorname{Ext}_{B}}^{1}\left(S_{j}, S_{j^{\prime}}\right)=1$ if there exists a cycle containing $i \longrightarrow j \longrightarrow j^{\prime}$ of $Q$ and no double arrow $i \longrightarrow j$, otherwise it is zero.

(iv) Assume that there are both arrows $i \longrightarrow j$ and $i \longrightarrow j^{\prime}$. Then we observe isomorphisms $\operatorname{Ext}_{B}^{1}\left(S_{j}, S_{j^{\prime}}\right) \simeq \operatorname{Ext}_{A}^{1}\left(X_{j}, X_{j^{\prime}}\right) \simeq \underline{\operatorname{Hom}}_{A}\left(X_{j}, \Omega^{-1} X_{j^{\prime}}\right)$. Since we have no non-zero homomorphism from $S_{i}$ to $\Omega^{-1} X_{j^{\prime}}$, any non-zero homomorphism $X_{j} \rightarrow \Omega^{-1} X_{j^{\prime}}$ is monomorphism. Therefore all projective homomorphisms from $X_{j}$ to $\Omega^{-1} X_{j^{\prime}}$ are zero, and so we see an equality $\underline{\operatorname{Hom}}_{A}\left(X_{j}, \Omega^{-1} X_{j^{\prime}}\right)=$ $\operatorname{Hom}_{A}\left(X_{j}, \Omega^{-1} X_{j^{\prime}}\right)$. Thus we obtain $\operatorname{dim} \operatorname{Ext}_{B}^{1}\left(S_{j}, S_{j^{\prime}}\right)$ as follows:

- In the case of $j \neq j^{\prime}$ :

$\begin{cases}1 & \text { if there is a cycle containing } i \longrightarrow j \longrightarrow i \longrightarrow j^{\prime} \text { of } Q ; \\ 1 & \text { if there is a cycle containing } i \longrightarrow j \longrightarrow j^{\prime} \text { of } Q ; \\ 0 & \text { otherwise. }\end{cases}$

- In the case of $j=j^{\prime}$ :

$$
\begin{cases}1 \quad \text { if there is a cycle } i \rightleftarrows j \text { or } C i \rightleftarrows j \text { of } Q \\ & \text { with multiplicity exactly greater than } 1 ; \\ 1 & \text { if there is a cycle containing } i \longrightarrow j \bigcirc \text { of } Q ; \\ 0 & \text { otherwise. }\end{cases}
$$

(2) (i) Assume that there is no arrow $i \longrightarrow j$.

(a) We have isomorphisms

$$
\operatorname{Ext}_{B}^{1}\left(S_{j}, S_{i}\right) \simeq \operatorname{Ext}_{A}^{1}\left(S_{j}, \Omega S_{i}\right) \simeq \underline{\operatorname{Hom}}_{A}\left(S_{j}, S_{i}\right)=0 .
$$


(b) We obtain isomorphisms

$$
\begin{aligned}
\operatorname{Ext}_{B}^{1}\left(S_{i}, S_{j}\right) & \simeq \operatorname{Ext}_{A}^{1}\left(\Omega S_{i}, S_{j}\right) \\
& \simeq \underline{\operatorname{Hom}}_{A}\left(\Omega^{2} S_{i}, S_{j}\right) \\
& =\operatorname{Hom}_{A}\left(\Omega^{2} S_{i}, S_{j}\right),
\end{aligned}
$$

which implies that $\operatorname{dim} \operatorname{Ext}_{B}^{1}\left(S_{i}, S_{j}\right)$ coincides with the number of paths $i \stackrel{\alpha}{\longrightarrow} h \stackrel{\beta}{\longrightarrow} j$ of $Q$ with $\alpha \beta \in I$.

(ii) Assume that there is an arrow $i \longrightarrow j$.

(a) We observe isomorphisms

$$
\begin{aligned}
\operatorname{Ext}_{B}^{1}\left(S_{j}, S_{i}\right) & \simeq \operatorname{Ext}_{A}^{1}\left(X_{j}, \Omega S_{i}\right) \\
& \simeq \underline{\operatorname{Hom}}_{A}\left(X_{j}, S_{i}\right) \\
& =\operatorname{Hom}_{A}\left(X_{j}, S_{i}\right),
\end{aligned}
$$

and so one sees that $\operatorname{dim} \operatorname{Ext}_{B}^{1}\left(S_{j}, S_{i}\right)=\operatorname{dim} \operatorname{Ext}_{A}^{1}\left(S_{i}, S_{j}\right)$.

(b) We have isomorphisms

$$
\begin{aligned}
\operatorname{Ext}_{B}^{1}\left(S_{i}, S_{j}\right) & \simeq \operatorname{Ext}_{A}^{1}\left(\Omega S_{i}, X_{j}\right) \\
& \simeq \underline{\operatorname{Hom}}_{A}\left(S_{i}, \Omega^{-2} X_{j}\right) \\
& =\operatorname{Hom}_{A}\left(S_{i}, \Omega^{-2} X_{j}\right) .
\end{aligned}
$$

Thus we get the following equalities.

(b1) In the case that $P_{j}$ is uniserial:

$$
\operatorname{dim} \operatorname{Ext}_{B}^{1}\left(S_{i}, S_{j}\right)=\left\{\begin{aligned}
& 2 \quad \text { if there is a path } h \stackrel{\beta}{\rightleftarrows} i \stackrel{\gamma}{\longrightarrow} j \text { of } Q \\
& \text { with } \alpha \beta \in I \text { and } \beta \gamma \notin I ; \\
& 1 \quad \text { otherwise. }
\end{aligned}\right.
$$

(b2) In the case that $P_{j}$ is non-uniserial: $\operatorname{dim} \operatorname{Ext}_{B}^{1}\left(S_{i}, S_{j}\right)$ is the number of paths $(\mathrm{b} 2-1) i \stackrel{\alpha}{\longrightarrow} h \stackrel{\beta}{\longrightarrow} j$ and $(\mathrm{b} 2-2) \quad h \stackrel{\beta}{\underset{\alpha}{\rightleftarrows}} i \stackrel{\gamma}{\longrightarrow} j$ with $\alpha \beta \in I$ and $\beta \gamma \notin I$.

(3) We have isomorphisms $\operatorname{Ext}_{B}^{1}\left(S_{i}, S_{i}\right) \simeq \operatorname{Ext}_{A}^{1}\left(\Omega S_{i}, \Omega S_{i}\right) \simeq \operatorname{Ext}_{A}^{1}\left(S_{i}, S_{i}\right)$.

6.3. Proof of our theorem. Our theorem is proved dividing into three cases as well as the definition of mutation of SB quivers.

6.3.1. Non-multiplex case. We show Theorem 3.1 in the case of non-multiplex. Assume that $A=k Q / I$ is a symmetric special biserial algebra and is non-multiplex at $i$. We use the notation of Definition 3.2 and Definition-Theorem 2.8.

Proof. (1) We first show that the quiver of $\mu_{i}^{+}(A)$ coincides with $\mu_{i}^{+}(Q, \mathrm{C})$.

(QM1) The calculations 6.2 (1)(ii) and (1)(iv) yield the new arrows of (QM1-1) and (QM1-2), respectively. 
(QM3) By 6.2 (2)(ii)(a), we reverse arrows $i \longrightarrow j$. Using 6.2 (2)(i)(b) and (2)(ii)(b2$1)$, we get the new arrows of (QM3-1). To obtain the new arrows of (QM3-2), we need to consider two cases: If $P_{j}$ is uniserial, then they occur by 6.2 (2)(ii)(b1). If $P_{j}$ is non-uniserial, then we have the new arrows of (QM3-2) by considering $h=j$ and $\gamma=\alpha$ in $6.2(2)(\mathrm{ii})(\mathrm{b} 2-2)$.

It is observed that the other arrows do not change.

(2) We show that the cycle-decomposition of $\mu_{i}^{+}(A)$ is given by Definition 3.3. Note that $\mu_{i}^{+}(A)$ is symmetric special biserial by Lemma 2.5 .

(QM1) We consider a new arrow $x$ as in (QM1). One defines a morphism $T_{j} \rightarrow T_{h}$ corresponding to $x$ as follows:

$$
x: P_{j} \stackrel{\beta}{\rightarrow} P_{i} \stackrel{\alpha}{\rightarrow} P_{h} \text { or } P_{j} \stackrel{\beta}{\rightarrow} P_{i} \stackrel{\gamma}{\rightarrow} P_{i} \stackrel{\alpha}{\rightarrow} P_{h} .
$$

(QM1-1) Assume that $h \neq j$. If an arrow $y$ satisfies $y \alpha \notin I$ or $\beta y \notin I$, then we obtain $C_{y}=C_{\alpha}$ or $C_{y}=C_{\beta}$. This implies that $y x \neq 0$ or $x y \neq 0$ in $\mu_{i}^{+}(A)$, which means that $C_{x}$ is given by replacing $\alpha \beta$ by $x$.

(QM1-2) Assume that $h=j, \beta \alpha \notin I$ and $\operatorname{mult}\left(C_{\alpha}\right)>1$. Then we can check $x^{2} \neq 0$ in $\mu_{i}^{+}(A)$, which implies that $C_{x}$ has only one arrow $x$.

(QM3) We consider new arrows $x$ and $y$ as in (QM3).

(QM3-1) In the case (QM3-1), we define morphisms $T_{i} \rightarrow T_{h}$ and $T_{j} \rightarrow T_{i}$ corresponding to $x$ and $y$, respectively:

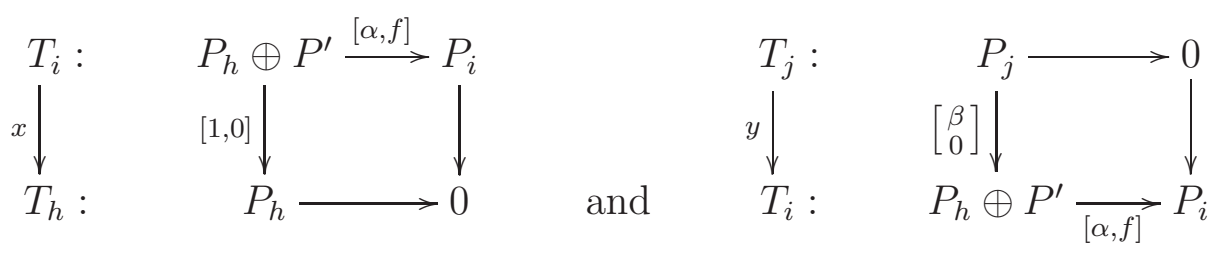

Then we see $x y=\beta$, which implies that $C_{x}=C_{y}$ and these are obtained by replacing $\beta$ in $C_{\beta}$ by $x y$.

(QM3-2) In the case (QM3-2), we have to consider two cases: One is that there exists an arrow $h \stackrel{\beta}{\longrightarrow} i$, and the other is that there is no such arrow. To avoid confusion, we write the arrow $\alpha$ of $\mu_{i}^{+}(Q, \mathrm{C})$ by $\alpha^{\prime}$.

(i) Assume that there exists an arrow $h \stackrel{\beta}{\longrightarrow} i$, and then we have arrows $i \underset{\beta}{\stackrel{\alpha}{\rightleftarrows}} h \stackrel{\gamma}{\longrightarrow} j$ of $Q$ with $\alpha \gamma \notin I$. Since $A$ is special biserial, we observe that $\alpha \beta$ belongs to $I$. By our assumption, we obtain that $\beta \alpha \notin I$. We define morphisms $T_{i} \rightarrow T_{h}$ and $T_{h} \rightarrow T_{i}$ corresponding to $x$ and $\alpha^{\prime}$ of $\mu_{i}^{+}(Q, \mathrm{C})$ as follows:
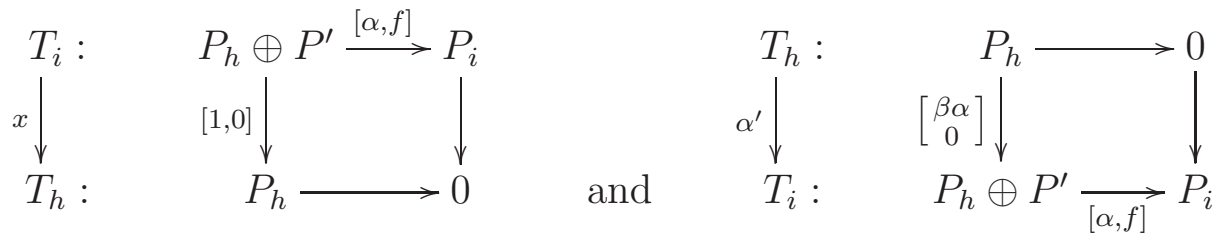
We can easily check $x \alpha^{\prime} \neq 0$ and $\alpha^{\prime} x=0$ in $\mu_{i}^{+}(A)$. Moreover, an arrow $\delta$ with $\delta \beta \notin I$ satisfies $\delta x \neq 0$ in $\mu_{i}^{+}(A)$. Thus we see that $C_{x}$ is given by replacing $\beta$ by $x$.

(ii) Assume that there is no such arrow $h \stackrel{\beta}{\longrightarrow} i$. Then $P_{h}$ is uniserial.

(a) If there is no loop at $i$ in $C_{\alpha}$, then we define morphisms $T_{i} \rightarrow T_{h}$ and $T_{h} \rightarrow T_{i}$ corresponding to $x$ and $\alpha^{\prime}$ of $\mu_{i}^{+}(Q, \mathrm{C})$ as follows:

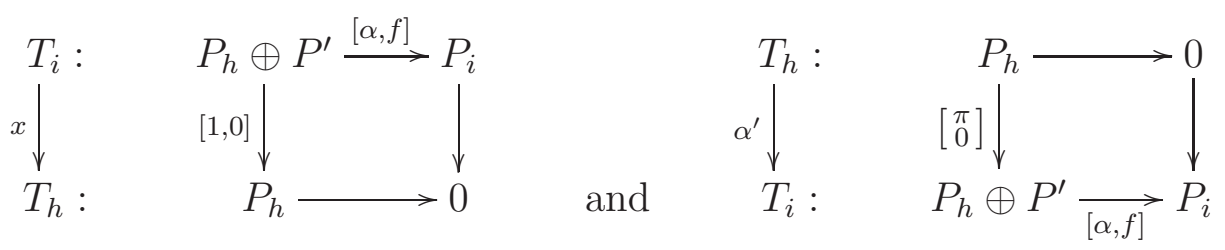

where $\pi$ is the composition of the canonical epimorphism $P_{h} \rightarrow S_{h}$ and the canonical inclusion $S_{h} \rightarrow P_{h}$. It is easy to see that $x \alpha^{\prime}=\pi \neq 0$. Let $\gamma$ be the unique arrow of $Q$ starting at $h$ with $\alpha \gamma \notin I$. We have $\alpha^{\prime} \gamma=0$ in $\mu_{i}^{+}(A)$. Since the number of arrows of $\mu_{i}^{+}(Q, \mathrm{C})$ starting at $h$ is at most two, we obtain $\alpha^{\prime} x \neq 0$. Thus, $C_{x}$ is a new cycle $i \underset{x}{\stackrel{\alpha^{\prime}}{\rightleftarrows}} h$.

(b) Assume that there is a loop $\beta$ at $i$ in $C_{\alpha}$. Then we observe that $\beta^{2} \in I$ and that the complex $T_{i}$ has of the form $P_{h} \oplus P_{h} \stackrel{[\alpha, \beta \alpha]}{\longrightarrow} P_{i}$. To avoid confusion, we write $\beta$ in $\mu_{i}^{+}(Q, \mathrm{C})$ by $\beta^{\prime}$. We define morphisms $T_{i} \rightarrow T_{h}, T_{h} \rightarrow T_{i}$ and $T_{i} \rightarrow T_{i}$ corresponding to $x, \alpha^{\prime}$ and $\beta^{\prime}$ as follows:
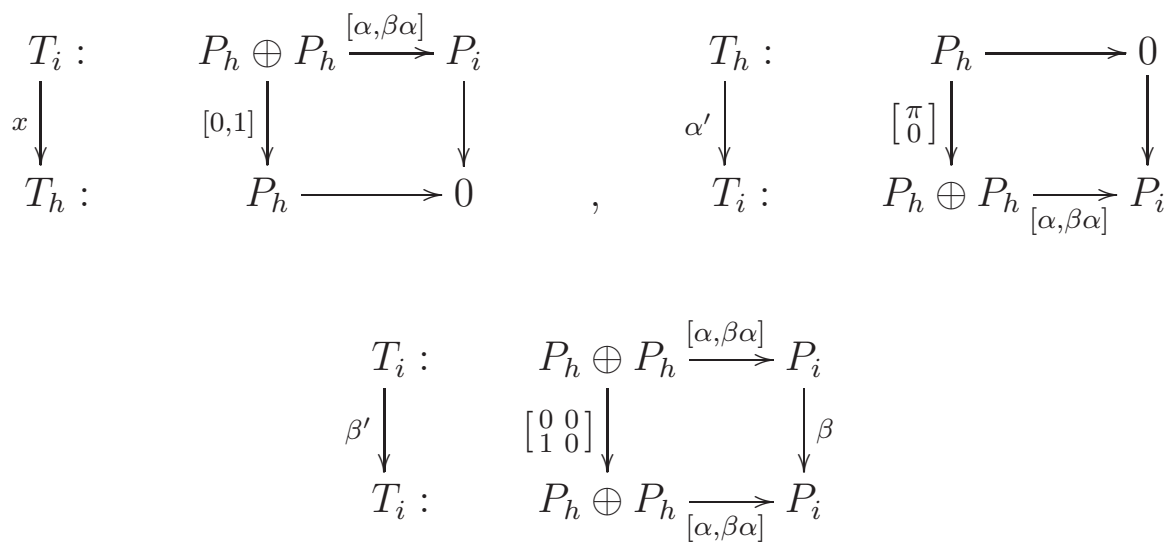

Then we get $x \beta^{\prime} \alpha^{\prime}=\pi$. It follows from the same argument above that $\alpha^{\prime} x \neq 0$ in $\mu_{i}^{+}(A)$. Thus we see that $C_{x}$ is a new cycle

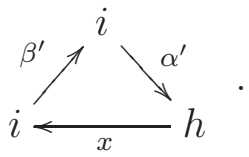

(3) Finally, we give the multiplicity of each cycle of $\mu_{i}^{+}(Q, \mathrm{C})$. Note that it is determined by the Cartan data of $\mu_{i}^{+}(A)$. To do this, we use the formula of Proposition 6.1. Since every indecomposable projective $A$-module $P_{j}$ distinct from $P_{i}$ is a direct summand of $T(i)$, the Cartan datum $\operatorname{dim} \operatorname{Hom}_{\mathrm{D}^{\mathrm{b}}(\bmod A)}\left(T_{h}, T_{j}\right)$ for $h \neq i$ and $j \neq i$ is equal to $\operatorname{dim} \operatorname{Hom}_{A}\left(P_{h}, P_{j}\right)$. Thus, we have only to show that the multiplicity of a cycle as in Definition $3.3(2)$ (ii)(b) is 1 . Put $m:=\operatorname{mult}\left(C_{\alpha}\right)$. 
Assume that there is no loop at $i$ in $C_{\alpha}$. Let $\beta: i \longrightarrow j$ be an arrow of $Q$ distinct from $\alpha$ if it exists. Then the complex $T_{i}$ has of the form $P_{h} \oplus P_{j} \stackrel{[\alpha, \beta]}{\longrightarrow} P_{i}$ if $\beta$ exists, otherwise $T_{h} \stackrel{\alpha}{\rightarrow} P_{i}$.

- If $\beta$ exists and $C_{\beta}=C_{\alpha}$, then we have equalities

$$
\begin{aligned}
\operatorname{dim} \operatorname{Hom}_{\mathrm{D}^{\mathrm{b}}(\bmod A)}\left(T_{h}, T_{i}\right)= & \operatorname{dim} \operatorname{Hom}_{A}\left(P_{h}, P_{h}\right)+\operatorname{dim} \operatorname{Hom}_{A}\left(P_{h}, P_{j}\right) \\
& -\operatorname{dim} \operatorname{Hom}_{A}\left(P_{h}, P_{i}\right) \\
= & (m+1)+m-2 m \\
= & 1 .
\end{aligned}
$$

- Otherwise,

$$
\begin{aligned}
& \operatorname{dim} \operatorname{Hom}_{\mathrm{D}^{\mathrm{b}}(\bmod A)}\left(T_{h}, T_{i}\right)=\operatorname{dim}_{\operatorname{Hom}_{A}}\left(P_{h}, P_{h}\right)-\operatorname{dim} \operatorname{Hom}_{A}\left(P_{h}, P_{i}\right) \\
& =(m+1)-m \\
& =1 \text {. }
\end{aligned}
$$

If there is a loop $\beta$ at $i$ of $Q$ in $C_{\alpha}$, then the complex $T_{i}$ has of the form $P_{h} \oplus P_{h} \stackrel{[\alpha, \beta \alpha]}{\longrightarrow} P_{i}$. We obtain equalities

$$
\begin{aligned}
\operatorname{dim} \operatorname{Hom}_{\mathrm{D}^{\mathrm{b}}(\bmod A)}\left(T_{h}, T_{i}\right) & =2 \cdot \operatorname{dim} \operatorname{Hom}_{A}\left(P_{h}, P_{h}\right)-\operatorname{dim} \operatorname{Hom}_{A}\left(P_{h}, P_{i}\right) \\
& =2(m+1)-2 m \\
& =2 .
\end{aligned}
$$

These give that the multiplicity of our cycle is 1 .

6.3.2. Multiplex case (1). We prove Theorem 3.1 in the case of multiplex. Assume that $A=k Q / I$ is a symmetric special biserial algebra and is multiplex at $i$. We use the notation of Definition 3.5 and Definition-Theorem 2.8. Suppose that $j^{\prime} \neq h$.

Proof. (1) We first show that the quiver of $\mu_{i}^{+}(A)$ coincides with $\mu_{i}^{+}(Q, \mathrm{C})$.

(QM1)' The calculations 6.2 (1)(ii) and (1)(iv) yield the new arrows of (QM1-1)' and (QM1-2)', respectively. However, 6.2 (1)(iv) says that there is no new arrow from $j$ to $h$.

(QM2)' The vertex $j$ belongs to exactly two cycles: One is $C_{\alpha}$ and the other is $C_{\beta}$. By 6.2 (2)(ii)(b2), we have no arrow from $i$ to $j$, which means that we remove $\alpha$. If $j^{\prime} \neq j$, by $6.2(2)(\mathrm{i})(\mathrm{a})$, there is no new arrow from $j^{\prime}$ to $i$. If $j^{\prime}=j$, by 6.2 (2)(ii)(a) and $h \neq j$, the number of new arrows from $j$ to $i$ is 1 . Therefore we remove $\alpha^{\prime}$.

(QM3)' The new arrows of (QM3-1)' are obtained by the same argument in the proof to non-multiplex case. We consider new arrows of (QM3-2)', which occurs if $P_{h}$ is uniserial: Note that $h \neq j$. It follows from 6.2 (2)(ii)(b1) that the number of arrows from $i$ to $h$ is exactly two: One is $\beta^{\prime}$ and the other is a new arrow $y$. By 6.2 (2)(ii)(a), we have exactly one arrow from $h$ to $i$, which is a new arrow $x$.

It is observed that the other arrows do not change.

(2) We show that the cycle-decomposition of $\mu_{i}^{+}(A)$ is given by Definition 3.6. Note that $\mu_{i}^{+}(A)$ is symmetric special biserial by Lemma 2.5. By considering the same argument in the proof to non-multiplex case, we have only to give morphisms among the direct 
summands of $T$ corresponding to $\beta, \beta^{\prime}$ and $x, y$ of (QM3-2)'. Note that $T_{i}$ has of the form $P_{j} \oplus P_{h} \stackrel{\left[\alpha, \beta^{\prime}\right]}{\longrightarrow} P_{i}$.

To avoid confusion, we write the arrows $\beta$ and $\beta^{\prime}$ of $\mu_{i}^{+}(Q, \mathrm{C})$ by $\beta_{*}$ and $\beta_{*}^{\prime}$, respectively. We define morphisms of $T_{i} \rightarrow T_{j}$ and $T_{h} \rightarrow T_{i}$ corresponding to $\beta_{*}$ and $\beta_{*}^{\prime}$ as follows:

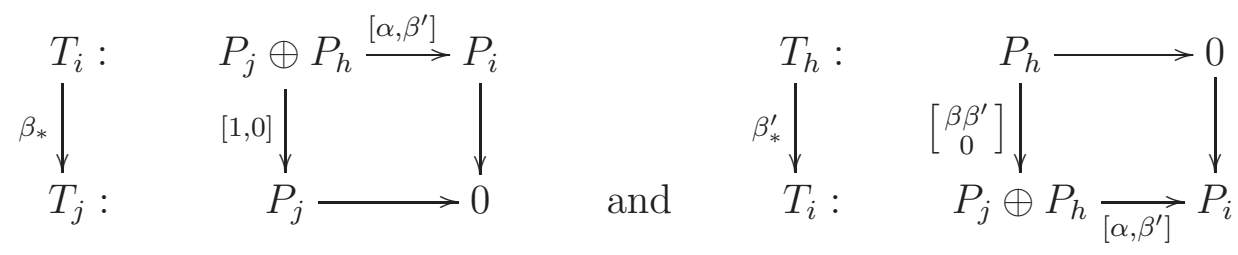

Assume that $P_{h}$ is uniserial. Then we define morphisms $T_{i} \rightarrow T_{h}$ and $T_{h} \rightarrow T_{i}$ corresponding to $x$ and $y$ as follows:

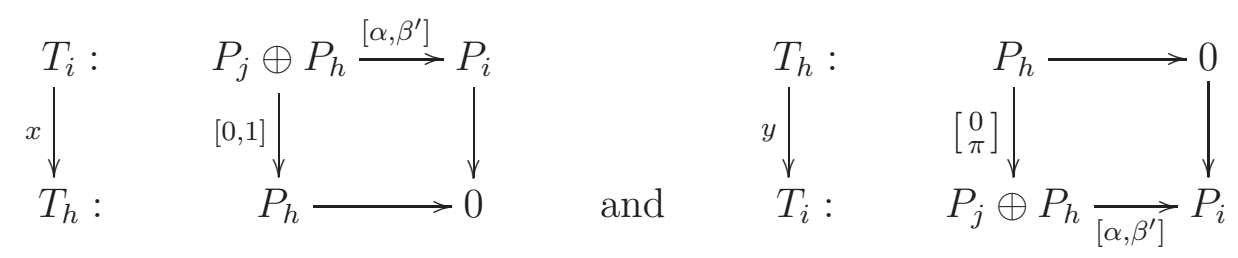

(3) We get the multiplicity of each cycle by the same argument in the proof to nonmultiplex case.

Thus the proof is complete.

6.3.3. Multiplex case (2). Finally we have to show Theorem 3.1 in the case of subsection 3.3 . However it follows from the same argument in the proof to non-multiplex and multiplex cases.

\section{REFERENCES}

[AIR] T. Adachi; O. Iyama; I. Reiten, $\tau$-tilting theory. Compos. Math. 150 (2014), no. 3, 415-452.

[A] T. Ainara, Mutating Brauer tree. Math. J. Okayama Univ. 56 (2014), 1-16.

[AI] T. Ainara; O. IYama, Silting mutation in triangulated categories. J. Lond. Math. Soc. (2) 85 (2012), no. 3, 633-668.

[APR] M. Auslander; M. I. Platzeck; I. Reiten, Coxeter functors without diagrams. Trans. Amer. Math. Soc. 250 (1979), 1-46.

[Al] D. Avella-Alaminos, Derived classification of gentle algebras with two cycles. Bol. Soc. Mat. Mexicana (3) 14 (2008), no. 2, 177-216.

[Alp] J. L. AlPerin, Local representation theory. Cambridge University Press, Cambredge, 1986.

[An] M. A. Antipov, Derived equivalence of symmetric special biserial algebras. Zap. Nauchn. Sem. S.-PeterBurg. Otdel. Mat. Inst. Steklov. (POMI) 343 (2007), Vopr. Teor. Predts. Algebr. i Grupp. 15, 5-32, 272; translation in J. Math. Sci. (N.Y.) 147 (2007), no. 5, 6981-6994.

[BGP] I. N. BernšteǏn; I. M. Gelfand; V.A. Ponomarev, Coxeter functors, and Gabrial's theorem. Uspehi Mat. Nauk 28 (1973), no. 2 (170), 19-33.

[BHS] R. Bocian; T. Holm; A. Skowroński, Derived equivalence classification of one-parametric self-injective algebras. J. Pure Appl. Algebra 207 (2006), 491-536.

[BR] M.C.R. Butler; C.M. Ringel, Auslander-Reiten sequences with few middle terms and applications to string algebras. Comm. Algebra 15 (1987), no.1-2, 145-179.

[E] K. ERdmann, Blocks of tame representation type and related algebras. Lecture Note in Mathematics, 1428. Springer, 1990.

[FST] S. Fomin; M. Shapiro; D. Thurston, Cluster algebras and triangulated surface. I. Cluster complexes. Acta Math. 201 (2008), no. 1, 83-146. 
[FZ] S. Fomin; A. Zelevinsky, Cluster algebras. I. Foundations. J. Amer. Math. Soc. 15 (2002), no. $2,497-529$.

[GP] I. M. Gelfand; V.A. Ponomarev, Indecomposable representations of the Lorentz group. Uspehi Mat. Nauk 23 1968, no. 2 (140), 3-60.

$[\mathrm{H}]$ D. HAPPEL, Triangulated categories in the representation theory of finite-dimensional algebras. London Mathematical Society Lecture Note Series, 119. Cambridge University Press, Cambridge, 1988.

[HU] D. Happel; L. Unger, On a partial order of tilting modules. Algebr. Represent. Theory 8 (2005), no. $2,147-156$.

[K] M. Kauer, Derived equivalence of graph algebras. Trends in the representation theory of finitedimensional algebras (Seattle, WA, 1997), 201-213, Contemp. Math., 229, Amer. Math. Soc., Providence, RI, 1998.

[KR] M. Kauer; K. W. Roggenkamp, Higher-dimensional orders, graph-orders, and derived equivalences. J. Pure App. Algebra 155 (2001), no. 2-3, 181-202.

[KZ] S. König; A. Zimmermann, Tilting selfinjective algebras and Gorenstein orders. Quart. J. Math. Oxford Ser. (2) 48 (1997), no. 191, 351-361.

[MS] R. J. MARsh; S. Schroll, The geometry of Brauer graph algebras and cluster mutations. arXiv (2013), http://arxiv.org/abs/1309.4239.

[M] F. H. Membrillo-Hernández, Brauer tree algebras and derived equivalence. J. Pure Appl. Algebra 114 (1997) 231-258.

[O] T. OKuyama, Some examples of derived equivalent blocks of finite groups. unpublished paper (1998).

[P] Z. Pogorzaly, Algebras stably equivalent to selfinjective special biserial algebras. Comm. Algebra 22 (1994), 1127-1160.

[R1] J. RickARD, Morita theory for derived categories. J. London Math. Soc. (2) 39 (1989), no. 3, $436-456$.

[R2] J. Rickard, Derived categories and stable equivalence. J. Pure Appl. Algebra 61 (1989), 303-317.

[RS] C. Riedtmann; A. Schofield, On a simplicial complex associated with tilting modules. Comment. Math. Helv. 66 (1991), no. 1, 70-78.

[Ro] K. W. Roggenkamp, Biserial algebras and graphs. Algebras and modules, II (1996), 481-496, CMS Conf. Proc. 24, Amer. Math. Soc., providence, RI, 1998.

[SZ] J. SchröEr; A. Zimmermann, Stable endomorphism algebras of modules over special biserial algebras. Math. Z. 244 (2003), no. 3, 515-530.

[SW] A. Skowroński; J. Waschbüsch, Representation-finite biserial algebras. J. Reine Angew. Math. 345 (1983), 172-181.

[WW] B. Wald; J. Waschbüsch, Tame biserial algebras. J. Algebra 95 (1985), no. 2, 480-500.

Fakultät FÜr Mathematik, Universität Bielefeld, D-33501 Bielefeld, Germany

Current address: Graduate School of Mathematics, Nagoya University, Furocho, Chikusaku, Nagoya 464-8602, Japan

E-mail address: aihara.takuma@math.nagoya-u.ac.jp 